Pure and Applied Mathematics Quarterly

Volume 4, Number 4

(Special Issue: In honor of

Jean-Pierre Serre, Part 1 of 2)

$1165-1204,2008$

\title{
Intersection Numbers of Heegner Divisors on Shimura Curves
}

Kevin Keating and David P. Roberts

In foundational papers, Gross, Zagier, and Kohnen established two formulas for arithmetic intersection numbers of certain Heegner divisors on integral models of modular curves. In [GZ1], only one imaginary quadratic discriminant plays a role. In [GZ2] and [GKZ], two quadratic discriminants play a role. In this paper we generalize the two-discriminant formula from the modular curves $X_{0}(N)$ to certain Shimura curves defined over $\mathbb{Q}$.

Our intersection formula was stated in [Ro], but the proof was only outlined there. Independently, the general formula was given, in a weaker and less explicit form, in [Ke2]; there it was proved completely. This paper is thus a synthesis of parts of [Ro] and [Ke2]. The intersection multiplicities computed here were used in $[\mathrm{Ku}]$ to derive a relation between height pairings and special values of the derivatives of certain Eisenstein series. Two more recent related works are $[\mathrm{KR}]$, which concentrates on computing local intersection multiplicities at ramified primes under quite general hypotheses, and [KRY], which relates intersection numbers on Shimura curves to coefficients of modular forms. We note also that Zhang $[\mathrm{Zh}]$ has generalized all of [GZ1] from ground field $\mathbb{Q}$ to general totally real ground fields $F$, working with general Shimura curves. We expect that all of [GKZ] should generalize similarly.

We are happy to thank B. Gross and S. Kudla for their encouragement during the long period in which this work was carried out.

Received July 27, 2006. 


\section{EICHLER ORDERS}

Let $\Delta$ be a quaternion algebra over $\mathbb{Q}$ and let $p$ be a prime. We begin by defining Eichler orders in $\Delta \otimes \mathbb{Q}_{p}$. Let $\mathcal{E}_{p}$ be an order in $\Delta \otimes \mathbb{Q}_{p}$ and let $p^{e}$ be the reduced discriminant of $\mathcal{E}_{p}$. We say that $\mathcal{E}_{p}$ is an Eichler order of type $\left(p^{e}, 1\right)$ if $\mathcal{E}_{p}$ contains a subring isomorphic to $\mathbb{Z}_{p} \oplus \mathbb{Z}_{p}$. We say that $\mathcal{E}_{p}$ is an Eichler order of type $\left(1, p^{e}\right)$ if $\mathcal{E}_{p}$ contains a subring isomorphic to $\mathbb{Z}_{p^{2}}$, the ring of integers in the quadratic unramified extension of $\mathbb{Q}_{p}$. It is easily seen that two of these local Eichler orders are conjugate in $\Delta \otimes \mathbb{Q}_{p}$ if and only if they are of the same type. In fact, if $\mathcal{E}_{p}$ is an Eichler order of type $\left(p^{e}, 1\right)$ then $\Delta \otimes \mathbb{Q}_{p} \cong \mathbb{M}_{2}\left(\mathbb{Q}_{p}\right)$, and $\mathcal{E}_{p}$ is conjugate to the standard Eichler order

$$
\hat{\mathcal{O}}_{p^{e}, 1}=\left\{\left[\begin{array}{ll}
a & b \\
c & d
\end{array}\right] \in \mathbb{M}_{2}\left(\mathbb{Z}_{p}\right): p^{e} \mid c\right\} .
$$

Let $\mathcal{A}_{p}$ be a maximal order in $\Delta \otimes \mathbb{Q}_{p}$, and let $i: \mathbb{Z}_{p^{2}} \rightarrow \mathcal{A}_{p}$ be an embedding. If $\mathcal{E}_{p}$ is an Eichler order of type $\left(1, p^{e}\right)$ then $\mathcal{E}_{p}$ is conjugate to the standard Eichler order

$$
\hat{\mathcal{O}}_{1, p^{e}}=i\left(\mathbb{Z}_{p^{2}}\right)+p^{f} \mathcal{A}_{p},
$$

where $f=\lfloor e / 2\rfloor$. Note that if $e$ is even then $\Delta \otimes \mathbb{Q}_{p} \cong \mathbb{M}_{2}\left(\mathbb{Q}_{p}\right)$, while if $e$ is odd then $\Delta \otimes \mathbb{Q}_{p}$ is a division ring.

We also need to define the notion of an orientation on a local Eichler order $\mathcal{E}_{p}$, which we assume is not of type $(1,1)$. For $e \geq 1$ define rings $R_{p^{e}, 1}=\left(\mathbb{Z} / p^{e} \mathbb{Z}\right) \oplus$ $\left(\mathbb{Z} / p^{e} \mathbb{Z}\right)$ and $R_{1, p^{e}}=\mathbb{Z}_{p^{2}} / p^{e} \mathbb{Z}_{p^{2}}$. If $\mathcal{E}_{p}$ is an Eichler order of type $\left(p^{e}, 1\right)$ then an orientation on $\mathcal{E}_{p}$ is defined to be a ring homomorphism $\psi_{p}: \mathcal{E}_{p} \rightarrow R_{p^{e}, 1}$. If $\mathcal{E}_{p}$ is an Eichler order of type $\left(1, p^{e}\right)$ then an orientation on $\mathcal{E}_{p}$ is defined to be a ring homomorphism $\psi_{p}: \mathcal{E}_{p} \rightarrow R_{1, p^{e}}$. Thus if $\mathcal{E}_{p}$ is an Eichler order in $\Delta \otimes \mathbb{Q}_{p}$ which is not isomorphic to $\mathbb{M}_{2}\left(\mathbb{Z}_{p}\right)$ there are exactly two orientations on $\mathcal{E}_{p}$. The usefulness of giving orientations to our Eichler orders may be summarized in the statement that the automorphisms of the oriented order $\left(\mathcal{E}_{p}, \psi_{p}\right)$ are precisely the maps given by conjugation by elements of $\mathcal{E}_{p}^{\times}$.

To define global Eichler orders we let $N^{+}=\prod p^{n_{p}^{+}}$and $N^{-}=\prod p^{n_{p}^{-}}$be relatively prime positive integers and set $N=N^{+} N^{-}$. We say that an order $\mathcal{E}$ in $\Delta$ is an Eichler order of type $\left(N^{+}, N^{-}\right)$if $\mathcal{E} \otimes \mathbb{Z}_{p}$ is an Eichler order of type $\left(p^{n_{p}^{+}}, p^{n_{p}^{-}}\right)$for every prime $p$. An orientation on $\mathcal{E}$ consists of a collection $\left\{\psi_{p}\right\}_{p \mid N}$ of orientations on $\mathcal{E} \otimes \mathbb{Z}_{p}$ for every prime $p$ which divides $N$. 
Proposition 1.1. Let $\Delta$ be a quaternion algebra over $\mathbb{Q}$ and let $\left(N^{+}, N^{-}\right)$be relatively prime positive integers.

(a) $\Delta$ contains an Eichler order of type $\left(N^{+}, N^{-}\right)$if and only if $v_{p}\left(N^{-}\right)$is odd precisely for those primes $p$ which are ramified in $\Delta$.

(b) $\Delta$ contains only finitely many isomorphism classes of oriented Eichler orders of type $\left(N^{+}, N^{-}\right)$.

(c) If $\Delta$ is indefinite then $\Delta$ contains at most one isomorphism class of oriented Eichler orders of type $\left(N^{+}, N^{-}\right)$.

Proof: (a) It follows from the definitions that if $\Delta$ contains an Eichler order of type $\left(N^{+}, N^{-}\right)$then $v_{p}\left(N^{-}\right)$is odd if and only if $\Delta$ is ramified at $p$. On the other hand, if $N^{-}$satisfies this condition then one can easily construct an oriented Eichler order of type $\left(N^{+}, N^{-}\right)$from a maximal order in $\Delta$.

(b) Let $\left(\mathcal{E},\left\{\psi_{p}\right\}_{p \mid N}\right)$ be an oriented Eichler order in $\Delta$ of type $\left(N^{+}, N^{-}\right)$, let $\hat{\mathcal{E}}=\mathcal{E} \otimes \hat{\mathbb{Z}}$ be the profinite completion of $\mathcal{E}$, and let $\hat{\Delta}=\hat{\mathcal{E}} \otimes \mathbb{Q}$ be the ring of finite adèles of $\Delta$. Associated to each $\beta=\left(\beta_{p}\right) \in \hat{\Delta}^{\times}$there is a unique lattice $L_{\beta}$ in $\Delta$ such that $L_{\beta} \otimes \mathbb{Z}_{p}=\beta_{p}\left(\mathcal{E} \otimes \mathbb{Z}_{p}\right)$ for all primes $p$. There is a bijection between the double coset space $S=\Delta^{\times} \backslash \hat{\Delta}^{\times} / \hat{\mathcal{E}}^{\times}$and the set of all isomorphism classes of oriented Eichler orders of type $\left(N^{+}, N^{-}\right)$, which associates to $\beta \in \hat{\Delta}^{\times}$ the pair $\left(\mathcal{E}_{\beta},\left\{\phi_{p}^{\beta}\right\}_{p \mid N}\right)$, where $\mathcal{E}_{\beta}$ is the left order of $L_{\beta}$ and $\phi_{p}^{\beta}(x)=\psi_{p}\left(\beta_{p}^{-1} x \beta_{p}\right)$. Since $S$ is finite [Vi, III, Cor. 5.5], the claim follows.

(c) By the strong approximation theorem [Vi, III, Th. 4.3] the reduced norm $\mathrm{Nr}$ on $\hat{\Delta}^{\times}$induces a bijection between $S$ and the set $T=\mathbb{Q}^{\times} \backslash \hat{\mathbb{Q}}^{\times} / \operatorname{Nr}\left(\hat{\mathcal{E}}^{\times}\right)$. In fact $\operatorname{Nr}\left(\hat{\mathcal{E}}^{\times}\right)=\hat{\mathbb{Z}}^{\times}$, so $T$ has just one element.

Corollary 1.2. Let $\Delta$ be an indefinite quaternion algebra over $\mathbb{Q}$ ramified at the primes $p_{1}, p_{2}, \ldots, p_{s}$. Set $N^{-}=p_{1} p_{2} \cdots p_{s}$, and let $N^{+}$be a positive integer which is relatively prime to $N^{-}$. Then there are Eichler orders $\mathcal{O}_{1, N^{-}} \supset \mathcal{O}_{N^{+}, N^{-}}$ in $\Delta$ of types $\left(1, N^{-}\right)$and $\left(N^{+}, N^{-}\right)$such that $\mathcal{O}_{1, N^{-}} / \mathcal{O}_{N^{+}, N^{-}}$is a cyclic group of order $N^{+}$. The pair $\left(\mathcal{O}_{1, N^{-}}, \mathcal{O}_{N^{+}, N^{-}}\right)$is uniquely determined up to conjugacy in $\Delta$.

Proof: The existence of the pair $\left(\mathcal{O}_{1, N^{-}}, \mathcal{O}_{N^{+}, N^{-}}\right)$is clear; what must be proved is that all such pairs are conjugate in $\Delta$. Since $\Delta$ is indefinite, it follows from Proposition 1.1(c) that $\mathcal{O}_{1, N^{-}}$is determined uniquely up to conjugation. Let $\Sigma$ be the set of Eichler orders $\mathcal{E}$ of type $\left(N^{+}, N^{-}\right)$such that $\mathcal{E} \subset \mathcal{O}_{1, N^{-}}$and $\mathcal{O}_{1, N^{-}} / \mathcal{E}$ 
is cyclic of order $N^{+}$. For each prime $p$ such that $p \mid N^{+}$let $\Sigma_{p}$ denote the set of local Eichler orders $\mathcal{E}_{p}$ of type $\left(p^{n^{+}}, 1\right)$ such that $\mathcal{E}_{p} \subset \mathbb{M}_{2}\left(\mathbb{Z}_{p}\right)$ and $\mathbb{M}_{2}\left(\mathbb{Z}_{p}\right) / \mathcal{E}_{p}$ is cyclic. Then $\mathbb{S L}_{2}\left(\mathbb{Z}_{p}\right)$ acts transitively by conjugation on $\Sigma_{p}$. Therefore by the strong approximation theorem the group of elements of $\mathcal{O}_{1, N^{-}}^{\times}$with reduced norm 1 acts transitively by conjugation on $\Sigma$. It follows that the pair $\left(\mathcal{O}_{1, N^{-}}, \mathcal{O}_{N^{+}, N^{-}}\right)$ is determined uniquely up to conjugation in $\Delta$.

Let $D_{1}, D_{2}$ be negative integers which are squares $(\bmod 4)$ such that $\mathbb{Q}\left(\sqrt{D_{1}}\right) \nsucceq$ $\mathbb{Q}\left(\sqrt{D_{2}}\right)$. Let $n$ be an integer such that $n \equiv D_{1} D_{2}(\bmod 2)$ and let $B_{n}$ be the Clifford algebra of the binary quadratic form $q_{n}(x, y)=D_{1} x^{2}+2 n x y+D_{2} y^{2}$. Thus $B_{n}$ is a quaternion algebra over $\mathbb{Q}$ which is generated by elements $e_{1}, e_{2}$ such that $e_{j}^{2}=D_{j}$ for $j=1,2$ and $e_{1} e_{2}+e_{2} e_{1}=2 n$. Let $g_{j}=\left(D_{j}+e_{j}\right) / 2$ and let $S_{n}=\mathbb{Z}\left[g_{1}, g_{2}\right]$ be the subring of $B_{n}$ generated by $g_{1}$ and $g_{2}$. Then

$$
S_{n}=\mathbb{Z}+\mathbb{Z} g_{1}+\mathbb{Z} g_{2}+\mathbb{Z} g_{1} g_{2}
$$

is an order in $B_{n}$ with reduced discriminant $\delta_{n}=\left(n^{2}-D_{1} D_{2}\right) / 4$. We may view $S_{n}$ with the reduced norm form $\mathrm{Nr}$ as a quadratic space over $\mathbb{Z}$. By restricting Nr to $L_{n}=\mathbb{Z}+\mathbb{Z} g_{1}+\mathbb{Z} g_{2}$ we get a quadratic form

$$
Q_{n}(x, y, z)=\operatorname{Nr}\left(x+y g_{1}+z g_{2}\right)
$$

$$
=x^{2}+\frac{D_{1}^{2}-D_{1}}{4} y^{2}+\frac{D_{2}^{2}-D_{2}}{4} z^{2}+D_{1} x y+D_{2} x z+\frac{D_{1} D_{2}-n}{2} y z
$$

with determinant $2 \delta_{n}$.

Assume now that $n^{2}<D_{1} D_{2}$ and $\operatorname{gcd}\left(D_{1}, D_{2}\right)=1$. We factor the positive integer $-\delta_{n}$ into relatively prime factors $\delta_{n}^{+}, \delta_{n}^{-}$using the criterion

$$
\begin{aligned}
& p \mid \delta_{n}^{+} \text {if }\left(\frac{D_{j}}{p}\right)=+1 \text { for at least one } j=1,2, \\
& p \mid \delta_{n}^{-} \text {if }\left(\frac{D_{j}}{p}\right)=-1 \text { for at least one } j=1,2,
\end{aligned}
$$

where $\left(\frac{D_{j}}{p}\right)$ is the Kronecker symbol. Suppose $p \mid \delta_{n}$ and $p \nmid D_{1} D_{2}$. Then $D_{1} D_{2} \equiv n^{2}(\bmod 4 p)$, and hence $\left(\frac{D_{1}}{p}\right)=\left(\frac{D_{2}}{p}\right)$. Thus (1.6) and (1.7) uniquely determine the factorization $-\delta_{n}=\delta_{n}^{+} \delta_{n}^{-}$.

For $i=1,2$ let $\mathcal{O}_{D_{i}}=\mathbb{Z}\left[\left(D_{i}+\sqrt{D_{i}}\right) / 2\right]$ be the order of discriminant $D_{i}$ in $\mathbb{Q}\left(\sqrt{D_{i}}\right)$. For each prime $p$ we have $p \nmid D_{j}$ for at least one $j \in\{1,2\}$. Thus if 
$p \mid \delta_{n}^{+}$then $\mathcal{O}_{D_{j}} \otimes \mathbb{Z}_{p} \cong \mathbb{Z}_{p} \oplus \mathbb{Z}_{p}$, and if $p \mid \delta_{n}^{-}$then $\mathcal{O}_{D_{j}} \otimes \mathbb{Z}_{p} \cong \mathbb{Z}_{p^{2}}$. Since $S_{n}$ contains a subring isomorphic to $\mathcal{O}_{D_{j}}$, it follows that $S_{n}$ is an Eichler order of type $\left(\delta_{n}^{+}, \delta_{n}^{-}\right)$.

\section{HeEgner Divisors on Shimura CuRves}

Let $N^{+}, N^{-}$be positive integers such that $N^{-}=p_{1} p_{2} \ldots p_{s}$ is the product of an even number of distinct primes and $N=N^{+} N^{-}$is greater than 1 . In this section we construct a scheme $\mathcal{X}=\mathcal{X}_{N^{+}, N^{-}, m}$ associated to the triple $\left(N^{+}, N^{-}, m\right)$ for certain values of $m$. The scheme $\mathcal{X}$ is an integral model for a Shimura curve $X$ which is defined over $\mathbb{Q}$. We also define Heegner divisors $P_{D}$ on $X$ and $\mathcal{P}_{D}$ on $\mathcal{X}$, where $D$ is the discriminant of an order in an imaginary quadratic field.

The scheme $\mathcal{X}$ will be constructed as a moduli space for abelian surfaces $A$ with additional structure. Part of the additional structure on $A$ is a "special" $\mathcal{O}_{1, N^{--}}$ action, as defined by Drinfeld [Dr, $\S 2 \mathrm{~A}]$. Let $R$ be a ring, let $A$ be an abelian surface over $R$, and let $i: \mathcal{O}_{1, N^{-}} \rightarrow \operatorname{End}_{R}(A)$ be an embedding. For $a \in \mathcal{O}_{1, N^{-}}$ let $\operatorname{Tr}(a) \in \mathbb{Z}$ denote the reduced trace of $a$, and let $\tau(a)$ denote the image of $\operatorname{Tr}(a)$ under the natural map $\mathbb{Z} \rightarrow R$. The embedding $i: \mathcal{O}_{1, N^{-}} \rightarrow \operatorname{End}_{R}(A)$ is said to be special if the trace of the action of $i(a)$ on $\operatorname{Lie}(A)$ is equal to $\tau(a)$ for all $a \in \mathcal{O}_{1, N^{-}}$. (If all the primes $p_{1}, p_{2}, \ldots, p_{s}$ which ramify in $\Delta$ are invertible in $R$ then every embedding is special.) More generally, if $Y$ is a scheme and $A / Y$ is an abelian surface, we say that the embedding $i: \mathcal{O}_{1, N^{-}} \rightarrow \operatorname{End}_{Y}(A)$ is special if the induced map

$$
i_{R}: \mathcal{O}_{1, N^{-}} \longrightarrow \operatorname{End}_{R}\left(A \times_{Y} \operatorname{Spec}(R)\right)
$$

is special for every affine subscheme $\operatorname{Spec}(R)$ of $Y$.

We are interested in the moduli problem for isomorphism classes of triples $(A, i, Z)$ over a scheme $Y$, where $A / Y$ is an abelian surface, $i: \mathcal{O}_{1, N^{-}} \rightarrow \operatorname{End}_{Y}(A)$ is a special embedding, and $Z$ is a subgroup scheme of $A$ which is cyclic of order $N^{+}$in the sense of [KM, 1.4]. Since this moduli problem is not representable, we will add a level- $m$ structure to the problem for an appropriate value of $m$. The choice of $m$ depends on $N=N^{+} N^{-}$and on the imaginary quadratic discriminants $D_{1}, D_{2}$ which will be introduced in $\S 3$. Let $m$ be a positive integer which 
satisfies the following conditions:

$$
\begin{aligned}
& \operatorname{gcd}(m, N)=1, \\
& m=m_{1} m_{2} \text { for some } m_{1}, m_{2} \geq 4 \text { such that } \operatorname{gcd}\left(m_{1}, m_{2}\right)=1, \\
& p>D_{1} D_{2} / 4 N \text { for every prime } p \text { which divides } m .
\end{aligned}
$$

Fix an isomorphism $\mathcal{O}_{1, N^{-}} / m \mathcal{O}_{1, N^{-}} \cong \mathbb{M}_{2}(\mathbb{Z} / m \mathbb{Z})$. Then $i: \mathcal{O}_{1, N^{-}} \rightarrow \operatorname{End}_{Y}(A)$ induces a ring homomorphism

$$
i_{m}: \mathbb{M}_{2}(\mathbb{Z} / m \mathbb{Z}) \longrightarrow \operatorname{End}_{Y}(A[m]),
$$

where $A[m]$ denotes the $m$-torsion subgroup scheme of $A$. A $\Gamma_{1}(m)$-structure on the pair $(A, i)$ is defined to be a point $\beta$ in the kernel of $i_{m}\left(\left[\begin{array}{ll}1 & 0 \\ 0 & 0\end{array}\right]\right)$ which is defined over $Y$ and has exact order $m$ in the sense of [KM, 1.4].

Let $\mathcal{F}_{m}$ denote the functor from schemes to sets which associates to a scheme $Y$ the set of isomorphism classes of 4-tuples $(A, i, Z, \beta)$, where

(1) $A$ is an abelian surface defined over $Y$.

(2) $i: \mathcal{O}_{1, N^{-}} \rightarrow \operatorname{End}_{Y}(A)$ is a special embedding.

(3) $Z$ is a cyclic subgroup scheme of $A$ of order $N^{+}$which is defined over $Y$ and stabilized by $i\left(\mathcal{O}_{N^{+}, N^{-}}\right)$.

(4) $\beta$ is a $\Gamma_{1}(m)$-structure on $(A, i)$.

It follows from [Dr, Prop. 4.4] and [Bu, Lemma 2.2] that for $j=1,2$ the restriction of $\mathcal{F}_{m_{j}}$ to $\mathbb{Z}\left[1 / m_{j}\right]$-schemes is represented by a scheme over $\mathbb{Z}\left[1 / m_{j}\right]$. Therefore by $[\mathrm{KM}, 4.3 .4]$ the restriction of $\mathcal{F}_{m}$ to $\mathbb{Z}\left[1 / m_{j}\right]$-schemes is also represented by a $\mathbb{Z}\left[1 / m_{j}\right]$-scheme. It follows that the functor $\mathcal{F}_{m}$ is represented by a scheme $\mathcal{X}$. By [Dr, Prop. 4.4], the scheme $\mathcal{X} \otimes \mathbb{Z}[1 / m]$ is projective over $\mathbb{Z}[1 / m]$. Hence $X:=\mathcal{X} \otimes \mathbb{Q}$ is a projective curve over $\mathbb{Q}$.

Let $k$ be a field, let $x \in \mathcal{X}(k)$, and let $\left(A_{x}, i_{x}, Z_{x}, \beta_{x}\right)$ be the 4-tuple which corresponds to $x$. An endomorphism of the triple $\left(A_{x}, i_{x}, Z_{x}\right)$ is defined to be an endomorphism of $A_{x}$ which stabilizes $Z_{x}$ and commutes with $i_{x}(a)$ for every $a \in \mathcal{O}_{1, N^{-}}$. Let $D$ be a negative integer which is a square $(\bmod 4)$, let $K=$ $\mathbb{Q}(\sqrt{D})$, and let $\mathcal{O}_{D}=\mathbb{Z}[(D+\sqrt{D}) / 2]$ be the order of discriminant $D$ in $K$. It follows from Proposition 2.1 below that there are only finitely many $x \in X(\mathbb{C})$ such that $\operatorname{End}\left(A_{x}, i_{x}, Z_{x}\right) \cong \mathcal{O}_{D}$. Therefore we may define a divisor $Q_{D}$ on $X$ by setting $Q_{D}=\sum(x)$, where the sum is taken over all such $x$. It follows from the 
definition that $Q_{D}$ is defined over $\mathbb{Q}$. Write $D=c^{2} D_{0}$, where $c$ is the conductor of $\mathcal{O}_{D}$ and $D_{0}$ is the discriminant of $K$. Define a divisor $P_{D}$ on $X$ by setting $P_{D}=\sum_{b \mid c} Q_{b^{2} D_{0}}$. Then we have $P_{D}=\sum(x)$, where the sum is taken over points $x \in X(\mathbb{C})$ such that $\mathcal{O}_{D}$ embeds as a subring in $\operatorname{End}\left(A_{x}, i_{x}, Z_{x}\right)$. We call $P_{D}$ the Heegner divisor of discriminant $D$ on $X$. Since $P_{D}$ is defined over $\mathbb{Q}$, we can also express $P_{D}$ as a formal sum $P_{D}=\sum(y)$ of irreducible subschemes $y$ of $X$. Let $\mathcal{P}_{D}$ be the divisor on $\mathcal{X}$ obtained by replacing each subscheme in this sum by its closure $\bar{y}$ in $\mathcal{X}$.

The following proposition gives a stringent condition that $A$ must satisfy if $(A, i, Z)$ corresponds to a point in the support of $Q_{D}$.

Proposition 2.1. Let $x \in X(\mathbb{C})$ be a point in the support of $Q_{D}$. Let $\mathcal{R}$ be the smallest order in $K=\mathbb{Q}(\sqrt{D})$ which contains $\mathcal{O}_{D}$ and whose conductor is not divisible by any prime which is ramified in $\Delta$. Then over $\mathbb{C}$ we have $A_{x} \cong E_{1} \times E_{2}$, where $E_{1}$ and $E_{2}$ are elliptic curves such that $\operatorname{End}\left(E_{1}\right) \cong \operatorname{End}\left(E_{2}\right) \cong \mathcal{R}$.

Proof: $\operatorname{Since} \operatorname{End}\left(A_{x}, i_{x}, Z_{x}\right) \cong \mathcal{O}_{D}$ there is an embedding of $\Delta \otimes K \operatorname{into} \operatorname{End}\left(A_{x}\right) \otimes$ $\mathbb{Q}$. Therefore by [Oo, Prop. 6.1] we see that $K$ splits $\Delta$ and that

$$
\operatorname{End}\left(A_{x}\right) \otimes \mathbb{Q} \cong \Delta \otimes K \cong \mathbb{M}_{2}(K) .
$$

It follows that $\operatorname{End}\left(A_{x}\right)$ is isomorphic to an order $S$ in $\mathbb{M}_{2}(K)$, and that the complex points of $A_{x}$ may be identified with a quotient $\mathbb{C}^{2} / L$, where $L$ is a $\mathbb{Z}$ lattice in $K^{2} \subset \mathbb{C}^{2}$. The stabilizer of $L$ in $\mathbb{M}_{2}(K)$ is $S$, and hence for each prime $p$ the stabilizer of $L \otimes \mathbb{Z}_{p}$ in $\mathbb{M}_{2}\left(K \otimes \mathbb{Q}_{p}\right)$ is $S \otimes \mathbb{Z}_{p}$.

The homomorphism $i_{x}: \mathcal{O}_{1, N^{-}} \rightarrow \operatorname{End}\left(A_{x}\right) \cong S$ induces a map

$$
i_{x} \otimes \mathbb{Z}_{p}: \mathcal{O}_{1, N^{-}} \otimes \mathbb{Z}_{p} \longrightarrow S \otimes \mathbb{Z}_{p}
$$

If $p \nmid N^{-}$then $\mathcal{O}_{1, N^{-}} \otimes \mathbb{Z}_{p} \cong \mathbb{M}_{2}\left(\mathbb{Z}_{p}\right)$, and hence $S \otimes \mathbb{Z}_{p}$ is isomorphic to an order in $\mathbb{M}_{2}\left(K \otimes \mathbb{Q}_{p}\right)$ which contains $\mathbb{M}_{2}\left(\mathbb{Z}_{p}\right)$. Such an order must be isomorphic to $\mathbb{M}_{2}\left(\mathcal{R}_{p}\right)$ for some order $\mathcal{R}_{p}$ in $K \otimes \mathbb{Q}_{p}$. Since $\operatorname{End}\left(A_{x}, i_{x}, Z_{x}\right) \cong \mathcal{O}_{D}$ we have $\mathcal{R}_{p} \cong \mathcal{O}_{D} \otimes \mathbb{Z}_{p}$.

If $p \mid N^{-}$then $p$ is ramified in $\Delta$, and hence $\mathcal{O}_{1, N^{-}} \otimes \mathbb{Z}_{p} \cong \hat{\mathcal{O}}_{1, p}$. Since $K$ splits $\Delta$ we see that $K_{p}=K \otimes \mathbb{Q}_{p}$ is a field which is a quadratic extension of $\mathbb{Q}_{p}$. Let $\mathcal{O}_{K_{p}}=\mathcal{O}_{K} \otimes \mathbb{Z}_{p}$ be the ring of integers in $K_{p}$. We will show that $S \otimes \mathbb{Z}_{p} \cong \mathbb{M}_{2}\left(\mathcal{O}_{K_{p}}\right)$. Choose a $\mathbb{Q}_{p}$-embedding $\psi: K_{p} \rightarrow \Delta \otimes \mathbb{Q}_{p}$. We give $\Delta \otimes \mathbb{Q}_{p}$ the structure of a $K_{p}$-vector space by setting $a \cdot v=v \psi(a)$ for $a \in K_{p}$, 
$v \in \Delta \otimes \mathbb{Q}_{p}$. Left multiplication gives a representation of $\Delta \otimes \mathbb{Q}_{p}$ on this 2dimensional $K_{p}$-vector space. On the other hand, since $S \otimes \mathbb{Z}_{p}$ is isomorphic to an order in $\mathbb{M}_{2}\left(K_{p}\right)$, the map $i_{x} \otimes \mathbb{Q}_{p}$ induces a representation of $\Delta \otimes \mathbb{Q}_{p}$ on $K_{p}^{2}$. By the Skolem-Noether theorem these two representations are isomorphic. Let $\Phi: K_{p}^{2} \rightarrow \Delta \otimes \mathbb{Q}_{p}$ be a $\left(\Delta \otimes \mathbb{Q}_{p}\right)$-equivariant isomorphism of $K_{p}$-vector spaces. Since $\mathcal{O}_{1, N^{-}} \otimes \mathbb{Z}_{p} \cong \hat{\mathcal{O}}_{1, p}$ stabilizes $L \otimes \mathbb{Z}_{p}$, it stabilizes $\Phi\left(L \otimes \mathbb{Z}_{p}\right)$ as well. Therefore $\Phi\left(L \otimes \mathbb{Z}_{p}\right)$ is a left $\left(\mathcal{O}_{1, N^{-}} \otimes \mathbb{Z}_{p}\right)$-ideal, and hence also a right $\left(\mathcal{O}_{1, N^{-}} \otimes \mathbb{Z}_{p}\right)$-ideal. Since $\psi\left(\mathcal{O}_{K_{p}}\right) \subset \mathcal{O}_{1, N^{-}} \otimes \mathbb{Z}_{p}$ this implies that $\Phi\left(L \otimes \mathbb{Z}_{p}\right)$ is an $\mathcal{O}_{K_{p}}$-module. Since $\Phi\left(L \otimes \mathbb{Z}_{p}\right)$ is free of rank 4 over $\mathbb{Z}_{p}$, it is free of rank 2 over $\mathcal{O}_{K_{p}}$. Therefore $L \otimes \mathbb{Z}_{p}$ is also a free $\mathcal{O}_{K_{p}}$-module of rank 2. We conclude that the stabilizer $S \otimes \mathbb{Z}_{p}$ of $L \otimes \mathbb{Z}_{p}$ is isomorphic to $\mathbb{M}_{2}\left(\mathcal{O}_{K_{p}}\right)$.

So far we have proved that $\operatorname{End}\left(A_{x}\right)$ is isomorphic to an order $S$ in $\mathbb{M}_{2}(K)$ such that $S \otimes \mathbb{Z}_{p} \cong \mathbb{M}_{2}\left(\mathcal{R}_{p}\right)$ for all $p$, where $\mathcal{R}_{p}=\mathcal{O}_{K_{p}}$ if $p$ is ramified in $\Delta$ and $\mathcal{R}_{p}=\mathcal{O}_{D} \otimes \mathbb{Z}_{p}$ if $p$ is not ramified in $\Delta$. Hence the order $\mathcal{R}$ in the statement of the theorem is the unique order in $K$ such that $\mathcal{R} \otimes \mathbb{Z}_{p}=\mathcal{R}_{p}$ for all $p$. To complete the proof we will show that $S$ contains a nontrivial idempotent.

Let $L^{\prime} \supset L$ be the $\mathcal{O}_{K}$-lattice generated by $L$. By choosing a new $K$-basis for $K^{2} \subset \mathbb{C}^{2}$ we may assume that $L^{\prime}=\mathcal{O}_{K} \oplus \mathcal{I}$ for some ideal $\mathcal{I} \subset \mathcal{O}_{K}$. The ideal $\mathcal{I}$ may be chosen to be relatively prime to every $p$ such that $\mathcal{R}_{p}$ is not the maximal order in $K_{p}$. Let $A^{\prime}$ denote the abelian surface over $\mathbb{C}$ such that $A^{\prime}(\mathbb{C}) \cong \mathbb{C}^{2} / L^{\prime} \cong\left(\mathbb{C} / \mathcal{O}_{K}\right) \times(\mathbb{C} / \mathcal{I})$. The endomorphism ring of $A^{\prime}$ is

$$
\begin{aligned}
S^{\prime} & \cong \operatorname{End}_{\mathcal{O}_{K}}\left(L^{\prime}\right) \\
& \cong\left\{\left[\begin{array}{ll}
a & b \\
c & d
\end{array}\right]: a, d \in \mathcal{O}_{K}, b \in \mathcal{I}^{-1}, c \in \mathcal{I}\right\}
\end{aligned}
$$

which is a maximal order in $\mathbb{M}_{2}(K)$ containing $S$. There is an action of $\mathcal{O}_{1, N^{-}}$ on $A^{\prime}$ given by the map $i^{\prime}: \mathcal{O}_{1, N^{-}} \rightarrow S^{\prime}$ which is the composition of the inclusion $S \hookrightarrow S^{\prime}$ with $i_{x}: \mathcal{O}_{1, N^{-}} \rightarrow S$.

The inclusion $L \hookrightarrow L^{\prime}$ induces an $\mathcal{O}_{1, N^{-}}$-equivariant isogeny $\pi: A_{x} \rightarrow A^{\prime}$. The kernel of $\pi$ is a finite subgroup $G \cong L^{\prime} / L$ of $A_{x}(\mathbb{C})$ which is stabilized by $i\left(\mathcal{O}_{1, N^{-}}\right)$. Let $G \cong \oplus_{p} G_{p}$ be the decomposition of $G$ into its $p$-primary subgroups. Then $G_{p} \cong\left(L^{\prime} \otimes \mathbb{Z}_{p}\right) /\left(L \otimes \mathbb{Z}_{p}\right)$, and $G_{p}=\{0\}$ for all $p$ such that $\mathcal{R}_{p}=\mathcal{O}_{K} \otimes \mathbb{Z}_{p}$. In particular, $G_{p}=\{0\}$ if $p$ is ramified in $\Delta$. 
Let $p$ be a prime such that $G_{p} \neq\{0\}$. Since $S \otimes \mathbb{Z}_{p} \cong \mathbb{M}_{2}\left(\mathcal{R}_{p}\right)$ is the stabilizer of $L \otimes \mathbb{Z}_{p}$ in $\mathbb{M}_{2}\left(K \otimes \mathbb{Q}_{p}\right)$, we see that $L \otimes \mathbb{Z}_{p}$ is free of rank 2 over $\mathcal{R}_{p}$. Let $C_{p} \in \mathbb{M}_{2}\left(K \otimes \mathbb{Q}_{p}\right)$ be a matrix whose columns are $\mathcal{R}_{p}$-generators for $L \otimes \mathbb{Z}_{p}$. The columns of $C_{p}$ also serve as $\left(\mathcal{O}_{K} \otimes \mathbb{Z}_{p}\right)$-generators for $L^{\prime} \otimes \mathbb{Z}_{p}$, and by the assumption on $\mathcal{I}$ we have $L^{\prime} \otimes \mathbb{Z}_{p}=\left(\mathcal{O}_{K} \otimes \mathbb{Z}_{p}\right)^{2}$. Therefore $C_{p} \in \mathbb{G L}_{2}\left(\mathcal{O}_{K} \otimes \mathbb{Z}_{p}\right)$. Let $J=\left[\begin{array}{ll}1 & 0 \\ 0 & 0\end{array}\right]$. By multiplying one of the columns of $C_{p}$ by $1 / \operatorname{det}\left(C_{p}\right)$ we get a matrix $C_{p}^{\prime} \in \mathbb{S L}_{2}\left(\mathcal{O}_{K} \otimes \mathbb{Z}_{p}\right)$ such that $C_{p}^{\prime} J C_{p}^{\prime-1}=C_{p} J C_{p}^{-1}$ is a nontrivial idempotent which lies in $\operatorname{End}_{\mathcal{R}_{p}}\left(L \otimes \mathbb{Z}_{p}\right)=S \otimes \mathbb{Z}_{p}$.

For each $p$ such that $G_{p} \neq\{0\}$ choose $n_{p} \geq 1$ such that $p^{n_{p}}$ kills $G_{p}$. By the strong approximation theorem there exists a matrix $C \in \mathbb{S L}_{2}\left(\mathcal{O}_{K}\right)$ such that

$$
\begin{aligned}
& C \equiv C_{p}^{\prime} \quad\left(\bmod p^{n_{p}}\right) \text { for all } p \text { such that } G_{p} \neq\{0\} \\
& C \equiv I_{2} \quad(\bmod \mathcal{I}) .
\end{aligned}
$$

Then $e=C J C^{-1}$ is a nontrivial idempotent in $S$. Set $E_{1}=e A_{x}$ and $E_{2}=$ $(1-e) A_{x}$. Then $A_{x} \cong E_{1} \times E_{2}$ with $\operatorname{End}\left(E_{1}\right) \cong \operatorname{End}\left(E_{2}\right) \cong \mathcal{R}$.

Let $c$ be the conductor of $\mathcal{O}_{D}$.

Remark 2.2. Recall that the ray class field $K_{c}$ of $K$ with conductor $c \mathcal{O}_{K}$ is the maximum abelian extension of $K$ whose ramification conductor divides $c \mathcal{O}_{K}$. Let $x$ lie in the support of $Q_{D}$, so that $A_{x} \cong E_{1} \times E_{2}$. Since the elliptic curves $E_{1}$ and $E_{2}$ are defined over $K_{c}$, the 4 -tuple $\left(A_{x}, i_{x}, Z_{x}, \beta_{x}\right)$ is defined over $K_{c N^{+} m}$. Therefore $x$ is rational over $K_{c N^{+} m}$.

Remark 2.3. If $\operatorname{gcd}\left(c, N^{-}\right) \neq 1$ then by Proposition 2.1 we get $Q_{D}=0$. Therefore if $p$ divides $\operatorname{gcd}\left(c, N^{-}\right)$then $P_{D}=P_{D / p^{2}}$. Hence we may assume without loss of generality that $c$ is relatively prime to $N^{-}$.

Remark 2.4. Suppose $p^{t} \mid N^{+}$and $p$ is inert in $K$. Then the order in $K$ which stabilizes $Z \subset E_{1} \times E_{2}$ has conductor divisible by $p^{t}$. Hence if $p^{t} \nmid c$ then $P_{D}=0$.

Remark 2.5. Suppose $p \mid N^{-}$and $p$ splits in $K$. Then $\mathcal{O}_{1, N^{-}} \otimes \mathbb{Z}_{p} \cong \mathcal{O}_{1, p}$ cannot be embedded in $\mathbb{M}_{2}(K) \otimes \mathbb{Q}_{p} \cong \mathbb{M}_{2}\left(\mathbb{Q}_{p} \oplus \mathbb{Q}_{p}\right)$, so we have $P_{D}=0$.

Remark 2.6. Suppose $p^{2} \mid N^{+}$and $p$ is ramified in $K$. Then the order in $K$ which stabilizes $Z \subset E_{1} \times E_{2}$ has conductor divisible by $p$. Hence if $p \nmid c$ then $P_{D}=0$. 
To each point $x$ in the support of $P_{D}$ we will associate a collection of homomorphisms $\left\{\omega_{p}^{x}\right\}_{p \mid N}$ which is analogous to an orientation. The following well-known fact will be used to construct these homomorphisms.

Lemma 2.7. Let $R$ be a (possibly noncommutative) ring with 1 and let $M$ be a free left $R$-module of rank 1 generated by $e \in M$. For $\phi \in \operatorname{End}_{R}(M)$ define $f(\phi) \in R$ by the formula $\phi(e)=f(\phi)$. Then the map $f: \operatorname{End}_{R}(M) \rightarrow R^{o p}$ is an isomorphism of rings, uniquely determined by $M$ up to conjugation by units in $R^{o p}$.

Fix an orientation $\left\{\phi_{p}\right\}_{p \mid N}$ on $\mathcal{O}_{N^{+}, N^{-}}$and let $x \in X(\mathbb{C})$ be a point in the support of $P_{D}$. Let $p$ be a prime which divides $N$, let $T_{p}\left(A_{x}\right)$ be the $p$-adic Tate module of $A_{x}$, and let $U_{p}^{x} \supset T_{p}\left(A_{x}\right)$ be the lattice which corresponds to the $p$ primary subgroup of $Z_{x}$. It follows from [Re, Th. 18.7] that $T_{p}\left(A_{x}\right)$ is free of rank 1 over the maximal order $\mathcal{O}_{1, N^{-}} \otimes \mathbb{Z}_{p}$. The $\left(\mathcal{O}_{1, N^{-}} \otimes \mathbb{Z}_{p}\right)$-module structure $i_{x} \otimes \mathbb{Z}_{p}$ on $T_{p}\left(A_{x}\right)$ induces an $\left(\mathcal{O}_{N^{+}, N^{-}} \otimes \mathbb{Z}_{p}\right)$-module structure $i_{p}$ on $U_{p}^{x}$. If $p \mid N^{-}$then $U_{p}^{x}=T_{p}\left(A_{x}\right)$ is free of rank 1 as a left module over $\mathcal{O}_{N^{+}, N^{-}} \otimes \mathbb{Z}_{p}=\mathcal{O}_{1, N^{-}} \otimes \mathbb{Z}_{p}$. If $p \mid N^{+}$we may identify $\mathcal{O}_{1, N^{-}} \otimes \mathbb{Z}_{p}$ with $\mathbb{M}_{2}\left(\mathbb{Z}_{p}\right)$ and $\mathcal{O}_{N^{+}, N^{-}} \otimes \mathbb{Z}_{p}$ with the standard local Eichler order $\hat{\mathcal{O}}_{p^{n_{p}^{+}, 1}}$ defined in (1.1). Furthermore, there exists a generator $e$ for $T_{p}\left(A_{x}\right)$ over $\mathcal{O}_{1, N^{-}} \otimes \mathbb{Z}_{p} \cong \mathbb{M}_{2}\left(\mathbb{Z}_{p}\right)$ such that the cyclic group $U_{p}^{x} / T_{p}\left(A_{x}\right)$ is generated by $\left[\begin{array}{cc}p^{-n_{p}^{+}} & 0 \\ 0 & 1\end{array}\right] \cdot e+T_{p}\left(A_{x}\right)$. It follows that $U_{p}^{x}$ is free of $\operatorname{rank} 1$ over $\mathcal{O}_{N^{+}, N^{-}} \otimes \mathbb{Z}_{p} \cong \hat{\mathcal{O}}_{p^{n_{p}^{+}, 1}}$, with generator $\left[\begin{array}{cc}p^{-n_{p}^{+}} & 0 \\ 0 & 1\end{array}\right] \cdot e$.

Let $p$ be a prime which divides $N$. Since $U_{p}^{x}$ is free of rank 1 over $\mathcal{O}_{N^{+}, N^{-}} \otimes \mathbb{Z}_{p}$, by Lemma 2.7 we get a ring isomorphism

$$
\operatorname{End}\left(U_{p}^{x}, i_{p}\right) \longrightarrow \mathcal{O}_{N^{+}, N^{-}}^{o p} \otimes \mathbb{Z}_{p}
$$

It follows from the preceding paragraph that every $\mathbb{Z}_{p}$-endomorphism of $U_{p}^{x}$ which commutes with the image of $i_{p}$ stabilizes $T_{p}\left(A_{x}\right)$. Therefore

$$
\operatorname{End}\left(U_{p}^{x}, i_{p}\right) \cong \operatorname{End}\left(A_{x}, i_{x}, Z_{x}\right) \otimes \mathbb{Z}_{p}
$$

Using (2.12), (2.13), and the orientation $\phi_{p}$ on $\mathcal{O}_{N^{+}, N^{-}} \otimes \mathbb{Z}_{p}$ we get a homomorphism

$$
\omega_{p}^{x}: \operatorname{End}\left(A_{x}, i_{x}, Z_{x}\right) \otimes \mathbb{Z}_{p} \longrightarrow R_{p^{n_{p}^{+}, p^{n_{p}^{-}}}},
$$


where $n_{p}^{+}=v_{p}\left(N^{+}\right)$and $n_{p}^{-}=v_{p}\left(N^{-}\right)$.

Choose $D$ such that $P_{D} \neq 0$ and the conductor $c$ of $\mathcal{O}_{D}$ is relatively prime to $N$. We will use the homomorphisms $\left\{\omega_{p}^{x}\right\}_{p \mid N}$ to give sum decompositions of the divisors $P_{D}$ and $\mathcal{P}_{D}$. For each $x$ in the support of $P_{D}$ there are two embeddings of $\mathcal{O}_{D}$ into $\operatorname{End}\left(A_{x}, i_{x}, Z_{x}\right)$. Choose one of these and call it $\rho_{x}$. For $p \mid N, p \neq 2$

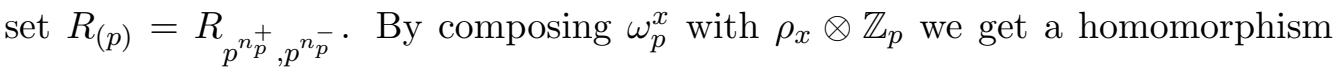
$\lambda_{p}^{x}: \mathcal{O}_{D} \otimes \mathbb{Z}_{p} \rightarrow R_{(p)}$. If $2 \mid N^{+}$set $R_{(2)}=R_{2^{n_{2}^{+}+1}, 1}$, while if $2 \mid N^{-}$set $R_{(2)}=R_{1,2^{n^{-}+1}}=R_{1,4}$. In either case there is a ring homomorphism

$$
\lambda_{2}^{x}: 1 \otimes \mathbb{Z}_{2}+2 \mathcal{O}_{D} \otimes \mathbb{Z}_{2} \longrightarrow R_{(2)}
$$

defined by

$$
\lambda_{2}^{x}(1 \otimes \alpha+2 \beta)=\alpha+2 \cdot \omega_{2}^{x} \circ\left(\rho_{x} \otimes \mathbb{Z}_{2}\right)(\beta) \quad\left(\bmod 2^{v_{2}(N)+1}\right)
$$

for $\alpha \in \mathbb{Z}_{2}, \beta \in \mathcal{O}_{D} \otimes \mathbb{Z}_{2}$. For every $p \mid N$ the ring homomorphism $\lambda_{p}^{x}$ is determined by the value of $\lambda_{p}^{x}(\sqrt{D})$. For each $p \mid N$ set $a_{p}=v_{p}(2 N)$. Then

$$
\lambda_{p}^{x}(\sqrt{D})^{2} \equiv D \quad\left(\bmod p^{a_{p}}\right) .
$$

If $2 \mid N$ then $\lambda_{2}^{x}(\sqrt{D})^{2}$ is well-defined modulo $2^{a_{2}+1}$, and it follows from (2.16) that

$$
\lambda_{2}^{x}(\sqrt{D})^{2} \equiv D \quad\left(\bmod 2^{a_{2}+1}\right) .
$$

Let $\iota_{p}$ denote the natural involution of the ring $R_{(p)}$. It follows from the definition of $\lambda_{p}^{x}$ that $\iota_{p}\left(\lambda_{p}^{x}(\sqrt{D})\right)=-\lambda_{p}^{x}(\sqrt{D})$.

For each $p \mid N$ let $b_{p}$ be an element of $R_{(p)}$ such that $b_{p}^{2} \equiv D\left(\bmod p^{a_{p}}\right)$ and $\iota_{p}\left(b_{p}\right)=-b_{p}$. If $2 \mid N$ assume further that $b_{2}^{2} \equiv D\left(\bmod 2^{a_{2}+1}\right)$. The assumption $P_{D} \neq 0$ implies that such $b_{p}$ exist; the assumption $p \nmid c$ and Remark 2.6 imply that there are at most two possibilities for $b_{p}$. Write $b=\left(b_{p}\right)_{p \mid N}$ and define

$$
V_{b}=\left\{x \in \operatorname{Supp}\left(P_{D}\right): \lambda_{p}^{x}(\sqrt{D})=b_{p} \text { for every } p \mid N\right\} .
$$

Then we get a divisor $P_{D, b}=\sum_{x \in V_{b}}(x)$ on $X$ such that $P_{D}=\sum_{b} P_{D, b}$. In general $P_{D, b}$ need not be defined over $\mathbb{Q}$ and may depend on the choices of the $\rho_{x}$, but the sum $P_{D, \pm b}=P_{D, b}+P_{D,-b}$ is a well-defined divisor over $\mathbb{Q}$. Define $\mathcal{P}_{D, \pm b}$ to be the closure of $P_{D, \pm b}$ in $\mathcal{X}$. Then $\mathcal{P}_{D, \pm b}$ is defined over $\mathbb{Z}$ and doesn't depend on the $\rho_{x}$. If $b=-b$ then $P_{D, \pm b}=2 P_{D, b}=2 P_{D}$ and hence $P_{D}=\frac{1}{2} P_{D, \pm b}$ and $\mathcal{P}_{D}=$ $\frac{1}{2} \mathcal{P}_{D, \pm b}$. Otherwise the divisors $P_{D}$ and $\mathcal{P}_{D}$ have natural sum decompositions $P_{D}=\sum P_{D, \pm b}$ and $\mathcal{P}_{D}=\sum \mathcal{P}_{D, \pm b}$. 


\section{INTERSECTION FORMULAS}

In this section we define the arithmetic intersection number $\left\langle\mathcal{Q}_{1} \cdot \mathcal{Q}_{2}\right\rangle_{\mathcal{X}}$ of two divisors $\mathcal{Q}_{1}, \mathcal{Q}_{2}$ on $\mathcal{X}$. We then give formulas for computing $\left\langle\mathcal{P}_{D_{1}, \pm b_{1}} \cdot \mathcal{P}_{D_{2}, \pm b_{2}}\right\rangle \mathcal{X}$ and $\left\langle\mathcal{P}_{D_{1}} \cdot \mathcal{P}_{D_{2}}\right\rangle_{\mathcal{X}}$ in certain cases.

We wish to define a $\mathbb{Q}$-linear pairing $\langle\cdot\rangle_{\mathcal{X}}$ of divisors on $\mathcal{X}$ which intersect properly on regular points of $\mathcal{X}$. It suffices to define $\left\langle T_{1} \cdot T_{2}\right\rangle_{\mathcal{X}}$ for dimension-1 subschemes $T_{1}, T_{2}$ of $\mathcal{X}$ whose intersection is supported on a finite set of closed points of $\mathcal{X}$, each of which is regular. In this case we have $T_{1} \cap T_{2} \cong \operatorname{Spec} R$ for some finite ring $R$, where $T_{1} \cap T_{2}$ is understood to mean $T_{1} \times \mathcal{X} T_{2}$. The arithmetic intersection number of $T_{1}$ with $T_{2}$ is defined to be $\left\langle T_{1} \cdot T_{2}\right\rangle_{\mathcal{X}}=\log \# R$. This formula extends by $\mathbb{Z}$-linearity to give pairings of divisors on $\mathcal{X}$.

The following proposition implies that the intersection of $\mathcal{P}_{D_{1}}$ and $\mathcal{P}_{D_{2}}$ is supported on a finite set of closed points of $\mathcal{X}$.

Proposition 3.1. Let $t$ be a point on $\mathcal{X}$ which lies in the support of the intersection of $\mathcal{P}_{D_{1}}$ with $\mathcal{P}_{D_{2}}$ and let $\left(A_{t}, i_{t}, Z_{t}, \beta_{t}\right)$ be the corresponding 4-tuple. Then $t$ is a closed point of characteristic $p>0$, and over $\overline{\mathbb{F}}_{p}$ we have $A_{t} \cong E \times E$ for any supersingular elliptic curve $E$.

Proof: Since $\mathbb{Q}\left(\sqrt{D_{1}}\right) \neq \mathbb{Q}\left(\sqrt{D_{2}}\right)$ the images of $\mathcal{O}_{D_{1}}$ and $\mathcal{O}_{D_{2}}$ generate a subalgebra of $\operatorname{End}\left(A_{t}, i_{t}, Z_{t}\right)$ with $\mathbb{Z}$-rank $\geq 4$. Therefore by [Oo, Prop.6.1], $t$ is a point of characteristic $p>0$, and $A_{t}$ is isogenous to the product of two supersingular elliptic curves. We have $t \in \bar{x}$ for some $x$ in the support of $P_{D_{1}}$, and it follows from Proposition 2.1 that $A_{x}$ is the product of two elliptic curves. Therefore $A_{t}$ is the product of two supersingular elliptic curves over $\overline{\mathbb{F}}_{p}$. A theorem of Deligne [Shi, Th.3.5] says that the isomorphism class of the product of two supersingular elliptic curves over $\overline{\mathbb{F}}_{p}$ does not depend on the factors. Therefore $A_{t} \cong E \times E$ for any supersingular elliptic curve $E$ over $\overline{\mathbb{F}}_{p}$. Finally, since $t$ is a point of characteristic $p$ lying in $\bar{x}, t$ is closed in $\mathcal{X}$. 
For $i=1,2$ let $D_{i}$ be a negative integer which is a square $(\bmod 4)$ and let $c_{i}$ be the conductor of the order $\mathcal{O}_{D_{i}}$. Assume that

$$
\begin{aligned}
& \mathbb{Q}\left(\sqrt{D_{1}}\right) \neq \mathbb{Q}\left(\sqrt{D_{2}}\right), \\
& l \nmid c_{1} c_{2} \text { for every prime } l \text { which divides } N, \\
& l \nmid \operatorname{gcd}\left(c_{1}, c_{2}\right) \text { for every prime } l \text { which divides } N .
\end{aligned}
$$

Assumption (3.2) guarantees that $P_{D_{1}}$ and $P_{D_{2}}$ are Heegner divisors in the sense of [Bi], and in any case may be made without loss of generality for $l \mid N^{-}$by Remark 2.3. Assumption (3.1) implies that $\mathcal{P}_{D_{1}}$ and $\mathcal{P}_{D_{2}}$ intersect properly on $\mathcal{X}$. Assumption (3.3) implies that every point in the support of the intersection of $\mathcal{P}_{D_{1}}$ with $\mathcal{P}_{D_{2}}$ is regular (see Corollary 6.3 ), and hence that $\left\langle\mathcal{P}_{D_{1}} \cdot \mathcal{P}_{D_{2}}\right\rangle_{\mathcal{X}}$ is defined.

Let $p$ be a finite prime. If $p$ is unramified in $\Delta$ let $\Delta(p)$ denote the quaternion algebra over $\mathbb{Q}$ which is ramified at $\infty, p, p_{1}, \ldots, p_{s}$. If $p=p_{j}$ is ramified in $\Delta$ let $\Delta(p)$ denote the quaternion algebra over $\mathbb{Q}$ which is ramified at $\infty, p_{1}, \ldots, p_{j-1}, p_{j+1}, \ldots, p_{s}$. Let $\mathcal{S}_{p}$ denote the set of Eichler orders $\mathcal{E} \subset \Delta(p)$ of type $\left(N^{+}, p N^{-}\right)$; it follows from Proposition 1.1(a) that $\mathcal{S}_{p}$ is not empty. We view the elements of $\mathcal{S}_{p}$ as lattices in $\Delta(p)$ with the $\mathbb{Z}$-valued quadratic forms induced by the reduced norm form on $\Delta(p)$. The assumption that $\mathcal{E}$ has type $\left(N^{+}, p N^{-}\right)$determines the isometry class of $\mathcal{E} \otimes \mathbb{Z}_{l}$ for every finite prime $l$, and $\mathcal{E} \otimes \mathbb{R} \cong \Delta(p) \otimes \mathbb{R}$ is positive definite since $\Delta(p)$ is ramified at $\infty$. Therefore every $\mathcal{E} \in \mathcal{S}_{p}$ belongs to the same genus of lattices in $\Delta(p)$. Let $\mathcal{G}_{p}$ be a set of representatives of the proper equivalence classes of this genus.

Let $n$ be an integer such that $n^{2}<D_{1} D_{2}$ and $n \equiv D_{1} D_{2}(\bmod 2)$. Let $L$ be a quadratic space over $\mathbb{Z}$ with finite rank and let $w_{L}$ denote the number of proper self-isometries of $L$. Define $\mathcal{R}_{L}\left(Q_{n}\right)$ to be the number of representations on $L$ of the quadratic form $Q_{n}$ defined in (1.4). Also let $r$ be the number of distinct prime divisors of $N$, and set

$$
\eta(m)=\frac{1}{2} \cdot m^{2} \cdot \prod_{p \mid m}\left(1-p^{-2}\right) .
$$

We now state the first version of our intersection formula.

Theorem 3.2. Let $D_{1}, D_{2}$ be negative integers which are squares (mod 4) and satisfy (3.1)-(3.3), and let $m$ be a positive integer which satisfies (2.2)-(2.4). 
Then the arithmetic intersection number of $\mathcal{P}_{D_{1}}$ with $\mathcal{P}_{D_{2}}$ on $\mathcal{X}$ is given by

$$
\left\langle\mathcal{P}_{D_{1}} \cdot \mathcal{P}_{D_{2}}\right\rangle_{\mathcal{X}}=2^{r-1} \cdot \eta(m) \cdot \sum_{p<\infty}\left(\sum_{\substack{n^{2}<D_{1} D_{2} \\ n^{2} \equiv D_{1} D_{2}(4 p N)}}\left(\sum_{L \in \mathcal{G}_{p}} \frac{\mathcal{R}_{L}\left(Q_{n}\right)}{w_{L}}\right) \cdot \alpha_{p}\left(Q_{n}\right)\right) \cdot \log p,
$$

where the local intersection multiplicities $\alpha_{p}\left(Q_{n}\right)$ are computed in (6.2) and (6.6).

Remark 3.3. The inner sum on the right side of (3.5) is a representation number in the sense of Siegel (see for instance [Ca, p. 377]).

Remark 3.4. Let $k$ be an algebraically closed field whose characteristic does not divide $m$, and let $(A, i)$ be an abelian surface with special $\mathcal{O}_{1, N^{-}}$-embedding defined over $k$. Then the pair $(A, i)$ admits $2 \eta(m)$ different $\Gamma_{1}(m)$-structures.

By strengthening assumption (3.3) we get a formula for $\left\langle\mathcal{P}_{D_{1}, \pm b_{1}} \cdot \mathcal{P}_{D_{2}, \pm b_{2}}\right\rangle \mathcal{X}$ which is stated explicitly in terms of finite Dirichlet series.

Definition 3.5. For $p$ prime and $e \geq 0$ define

$$
\begin{aligned}
& L_{p^{e}, 1}(s)=1+p^{-s}+p^{-2 s}+\cdots+p^{-e s}, \\
& L_{1, p^{e}}(s)=1-p^{-s}+p^{-2 s}-\cdots+(-1)^{e} p^{-e s} .
\end{aligned}
$$

For relatively prime positive integers $M^{+}, M^{-}$define

$$
L_{M^{+}, M^{-}}(s)=\prod_{p^{e} \| M^{+}} L_{p^{e}, 1}(s) \cdot \prod_{p^{e} \| M^{-}} L_{1, p^{e}}(s) .
$$

For each prime $p$ such that $p \mid N$ set $a_{p}=v_{p}(2 N)$. Since $\iota_{p}\left(\left(b_{j}\right)_{p}\right)=-\left(b_{j}\right)_{p}$ we have $\left(b_{1}\right)_{p}\left(b_{2}\right)_{p} \equiv h_{p}\left(\bmod p^{a_{p}}\right)$ for some $h_{p} \in \mathbb{Z}$. Let $h$ be an integer such that

$$
\begin{array}{lll}
h \equiv h_{p} & \left(\bmod p^{a_{p}}\right) & \text { for all } p \mid N, \\
h \equiv D_{1} D_{2} & (\bmod 2) & \text { if } 2 \nmid N .
\end{array}
$$

These congruences determine the class of $h(\bmod 2 N)$.

Theorem 3.6. Let $D_{1}, D_{2}$ be negative integers which are squares (mod 4) and satisfy (3.1)-(3.3), and let $m$ be a positive integer which satisfies (2.2)-(2.4). Assume further that $\operatorname{gcd}\left(D_{1}, D_{2}\right)=1$ and that $P_{D_{j}} \neq 0$ for $j=1,2$. Then the 
arithmetic intersection number of $\mathcal{P}_{D_{1}, \pm b_{1}}$ with $\mathcal{P}_{D_{2}, \pm b_{2}}$ on $\mathcal{X}$ is given by

$$
\left\langle\mathcal{P}_{D_{1}, \pm b_{1}} \cdot \mathcal{P}_{D_{2}, \pm b_{2}}\right\rangle_{\mathcal{X}}=2 \eta(m) \cdot \sum_{\substack{n^{2}<D_{1} D_{2} \\ n \equiv h(2 N)}} L_{\delta_{n}^{+} / N^{+}, \delta_{n}^{-} / N^{-}}^{\prime}(0),
$$

where $\eta(m)$ is given by (3.4), $h=h\left(b_{1}, b_{2}\right)$ is determined by (3.9) and (3.10), and $\delta_{n}^{+}, \delta_{n}^{-}$are determined by (1.6) and (1.7).

Remark 3.7. Suppose $p \mid N^{+}$. Then since $p \nmid c_{j}$ and $P_{D_{j}} \neq 0$, Remark 2.4 implies that $p$ is not inert in $\mathbb{Q}\left(\sqrt{D_{j}}\right)$. Similarly, Remark 2.5 implies that if $p \mid N^{-}$then $p$ is not split in $\mathbb{Q}\left(\sqrt{D_{j}}\right)$. Since $N \mid \delta_{n}$ for every $n$ such that $n \equiv h(\bmod 2 N)$, we get $N^{+} \mid \delta_{n}^{+}$and $N^{-} \mid \delta_{n}^{-}$. Hence the right side of (3.11) is well-defined.

For each value of $h(\bmod 2 N)$ such that $h^{2} \equiv D_{1} D_{2}(\bmod 4 N)$ there are $2^{r}$ pairs $\left(b_{1}, b_{2}\right)$ such that $\left(b_{1}\right)_{p}\left(b_{2}\right)_{p} \equiv h\left(\bmod p^{a_{p}}\right)$ for all $p \mid N$. Therefore by summing (3.11) over all $\left(b_{1}, b_{2}\right)$ we get a formula for $\left\langle\mathcal{P}_{D_{1}} \cdot \mathcal{P}_{D_{2}}\right\rangle$.

Corollary 3.8. Let $D_{1}, D_{2}$ be negative integers which are squares (mod 4) and satisfy (3.1)-(3.3), and let $m$ be a positive integer which satisfies (2.2)-(2.4). Assume further that $\operatorname{gcd}\left(D_{1}, D_{2}\right)=1$ and that $P_{D_{j}} \neq 0$ for $j=1,2$. Then

$$
\left\langle\mathcal{P}_{D_{1}} \cdot \mathcal{P}_{D_{2}}\right\rangle_{\mathcal{X}}=2^{r-1} \cdot \eta(m) \cdot \sum_{\substack{n^{2}<D_{1} D_{2} \\ n^{2} \equiv D_{1} D_{2}(4 N)}} L_{\delta_{n}^{+} / N^{+}, \delta_{n}^{-} / N^{-}}^{\prime}(0) .
$$

\section{INTERSECTION POINTS}

Let $t$ be a point in the support of the intersection of $\mathcal{P}_{D_{1}}$ with $\mathcal{P}_{D_{2}}$, and let $\left(A_{t}, i_{t}, Z_{t}, \beta_{t}\right)$ be the corresponding 4 -tuple. In this section we study the endomorphism ring of the triple $\left(A_{t}, i_{t}, Z_{t}\right)$. In particular, we show that $\operatorname{End}\left(A_{t}, i_{t}, Z_{t}\right)$ is an Eichler order, and we construct an orientation on $\operatorname{End}\left(A_{t}, i_{t}, Z_{t}\right)$ which is induced by the orientation $\left\{\phi_{l}\right\}_{l \mid N}$ on $\mathcal{O}_{N^{+}, N^{-}}$.

Let $T_{1}, T_{2}$ be dimension- 1 subschemes of $\mathcal{X}$ whose intersection is supported on a finite set of regular closed points of $\mathcal{X}$. Recall that the arithmetic intersection number of $T_{1}$ with $T_{2}$ is defined to be $\left\langle T_{1} \cdot T_{2}\right\rangle_{\mathcal{X}}=\log \# R$, where $T_{1} \cap T_{2} \cong \operatorname{Spec} R$. In practice, we will compute $\left\langle T_{1} \cdot T_{2}\right\rangle_{\mathcal{X}}$ as a sum

$$
\left\langle T_{1} \cdot T_{2}\right\rangle_{\mathcal{X}}=\sum_{p<\infty}\left(T_{1} \cdot T_{2}\right)_{p} \cdot \log p,
$$


where $\left(T_{1} \cdot T_{2}\right)_{p}$ is the intersection multiplicity of $T_{1}$ with $T_{2}$ at points of characteristic $p$. Thus $\left(T_{1} \cdot T_{2}\right)_{p}$ is equal to the length of the $\mathbb{Z}_{p}$-module $R \otimes \mathbb{Z}_{p}$. Let $W_{p}=W\left(\overline{\mathbb{F}}_{p}\right)$ denote the ring of Witt vectors with coefficients in $\overline{\mathbb{F}}_{p}$. Then $\left(T_{1} \cdot T_{2}\right)_{p}$ may also be computed as the length of the $W_{p}$-module $R \otimes W_{p}$, or as the intersection multiplicity of $T_{1} \otimes W_{p}$ with $T_{2} \otimes W_{p}$ on $\mathcal{X} \otimes W_{p}$.

Let $t$ be a point of characteristic $p$ in the support of the intersection of $\mathcal{P}_{D_{1}} \otimes W_{p}$ with $\mathcal{P}_{D_{2}} \otimes W_{p}$ on $\mathcal{X} \otimes W_{p}$. Then $t$ is defined over the residue field $\overline{\mathbb{F}}_{p}$ of $W_{p}$ and thus may be viewed as an element of $\mathcal{X}\left(\overline{\mathbb{F}}_{p}\right)$. Let $E$ be a supersingular elliptic curve over $\overline{\mathbb{F}}_{p}$ and set $\Lambda=\operatorname{End}(E)$. Then by Proposition 3.1 we have $A_{t} \cong E \times E$, and hence $\operatorname{End}\left(A_{t}\right) \cong \mathbb{M}_{2}(\Lambda)$. We will assume that $E$ is defined over $\mathbb{F}_{p}$; this implies that the elements of $\operatorname{End}(E)$ are defined over $\mathbb{F}_{p^{2}}$. It is well-known that $H_{p}=\Lambda \otimes \mathbb{Q}$ is a quaternion algebra over $\mathbb{Q}$ which is ramified at $p$ and $\infty$, and that $\Lambda$ is a maximal order in $H_{p}$. It follows from the definition of $\Delta(p)$ that there is an embedding $h: \Delta(p) \rightarrow \mathbb{M}_{2}\left(H_{p}\right)$ such that $h(\Delta(p))$ is the commutant of $i_{t}(\Delta)$ in $\mathbb{M}_{2}\left(H_{p}\right)$. The endomorphism ring of the triple $\left(A_{t}, i_{t}, Z_{t}\right)$ consists of those elements of $\mathbb{M}_{2}(\Lambda)$ which commute with every element of $i_{t}\left(\mathcal{O}_{1, N^{-}}\right)$and stabilize $Z_{t}$. Therefore $\operatorname{End}\left(A_{t}, i_{t}, Z_{t}\right)$ is identified via $h^{-1}$ with an order $\mathcal{E}$ in $\Delta(p)$.

We now determine the completions of $\operatorname{End}\left(A_{t}, i_{t}, Z_{t}\right) \cong \mathcal{E}$ at the finite places of $\mathbb{Q}$. Let $l \neq p$, let $T_{l}\left(A_{t}\right)$ denote the $l$-adic Tate module of $A_{t}$, and let $U_{l}^{t} \supset T_{l}\left(A_{t}\right)$ be the lattice which corresponds to the l-primary subgroup of $Z_{t}$. As in $\S 2, U_{l}^{t}$ is a free $\left(\mathcal{O}_{N^{+}, N^{-}} \otimes \mathbb{Z}_{l}\right)$-module of rank 1. Using Lemma 2.7 we get an isomorphism

$$
\operatorname{End}\left(A_{t}, i_{t}, Z_{t}\right) \otimes \mathbb{Z}_{l} \cong \mathcal{O}_{N^{+}, N^{-}}^{o p} \otimes \mathbb{Z}_{l} .
$$

Therefore if $l \neq p$ then $\operatorname{End}\left(A_{t}, i_{t}, Z_{t}\right) \otimes \mathbb{Z}_{l}$ is a local Eichler order of type $\left(l^{n_{l}^{+}}, l^{n_{l}^{-}}\right)$.

We now consider the completion of $\operatorname{End}\left(A_{t}, i_{t}, Z_{t}\right)$ at $p$. Leaving the subgroup $Z_{t}$ aside we see that $\operatorname{End}\left(A_{t}, i_{t}\right) \otimes \mathbb{Z}_{p}$ is the commutant of $i\left(\mathcal{O}_{1, N^{-}}\right) \otimes \mathbb{Z}_{p}$ in $\operatorname{End}\left(A_{t}\right) \otimes \mathbb{Z}_{p} \cong \mathbb{M}_{2}\left(\hat{\mathcal{O}}_{1, p}\right)$. If $p \nmid N^{-}$then $i_{t}\left(\mathcal{O}_{1, N^{-}}\right) \otimes \mathbb{Z}_{p} \cong \mathbb{M}_{2}\left(\mathbb{Z}_{p}\right)$ stabilizes $\hat{\mathcal{O}}_{1, p} \oplus \hat{\mathcal{O}}_{1, p}$, and hence $i_{t}\left(\mathcal{O}_{1, N^{-}}\right) \otimes \mathbb{Z}_{p}=g \mathbb{M}_{2}\left(\mathbb{Z}_{p}\right) g^{-1}$ for some $g \in \mathbb{G L}_{2}\left(\hat{\mathcal{O}}_{1, p}\right)$. Since $g \mathbb{M}_{2}\left(\hat{\mathcal{O}}_{1, p}\right) g^{-1}=\mathbb{M}_{2}\left(\hat{\mathcal{O}}_{1, p}\right)$ and the commutant of $\mathbb{M}_{2}\left(\mathbb{Z}_{p}\right)$ in $\mathbb{M}_{2}\left(\hat{\mathcal{O}}_{1, p}\right)$ is $\hat{\mathcal{O}}_{1, p} \cdot I_{2}$, it follows that

$$
\operatorname{End}\left(A_{t}, i_{t}\right) \otimes \mathbb{Z}_{p} \cong g\left(\hat{\mathcal{O}}_{1, p} \cdot I_{2}\right) g^{-1} \cong \hat{\mathcal{O}}_{1, p} .
$$

Thus if $p \nmid N$ then $\operatorname{End}\left(A_{t}, i_{t}, Z_{t}\right) \otimes \mathbb{Z}_{p} \cong \hat{\mathcal{O}}_{1, p}$. If $p \mid N^{+}$we may assume that $p \nmid D_{1}$ by (3.3). Then Remark 2.4 implies that $p$ is split in $\mathbb{Q}\left(\sqrt{D_{1}}\right)$, which contradicts the fact that $\mathcal{O}_{D_{1}} \otimes \mathbb{Z}_{p}$ embeds in $\operatorname{End}\left(A_{t}, i_{t}, Z_{t}\right) \otimes \mathbb{Z}_{p} \cong \hat{\mathcal{O}}_{1, p}$. So 
in fact (3.3) implies that there are no intersection points in characteristic $p$ if $p \mid N^{+}$.

Finally, if $p \mid N^{-}$we need to consider embeddings of $\mathcal{O}_{1, N^{-}} \otimes \mathbb{Z}_{p} \cong \hat{\mathcal{O}}_{1, p}$ into $\mathbb{M}_{2}\left(\hat{\mathcal{O}}_{1, p}\right)$ which are induced by special embeddings $i_{t}: \mathcal{O}_{1, N^{-}} \rightarrow \operatorname{End}\left(A_{t}\right)$. We can write $\hat{\mathcal{O}}_{1, p}=\mathbb{Z}_{p^{2}}+\mathbb{Z}_{p^{2}} \pi$, where $\mathbb{Z}_{p^{2}} \subset \hat{\mathcal{O}}_{1, p}$ is the ring of integers in an unramified quadratic extension of $\mathbb{Q}_{p}$, and $\pi$ is an element of $\hat{\mathcal{O}}_{1, p}$ which normalizes $\mathbb{Z}_{p^{2}}$ and satisfies $\pi^{2}=p$. Consider first the embedding of $\mathbb{Z}_{p^{2}}$ into $\mathbb{M}_{2}\left(\hat{\mathcal{O}}_{1, p}\right)$. It is not hard to show that since $i_{t}$ is special this embedding is conjugate by an element of $\mathbb{G L}_{2}\left(\hat{\mathcal{O}}_{1, p}\right)$ to the map

$$
x \longmapsto\left[\begin{array}{cc}
x & 0 \\
0 & x^{\prime}
\end{array}\right],
$$

where $x^{\prime}=\pi x \pi^{-1}$ is the Galois conjugate of $x$ over $\mathbb{Q}_{p}$. The commutant in $\mathbb{M}_{2}\left(\hat{\mathcal{O}}_{1, p}\right)$ of the image of $(4.4)$ is

$$
C=\left\{\left[\begin{array}{cc}
a & b \pi \\
c \pi & d
\end{array}\right]: a, b, c, d \in \mathbb{Z}_{p^{2}}\right\} .
$$

The image of $\pi$ under $i_{t} \otimes \mathbb{Z}_{p}$ is a matrix of the form

$$
\Pi=\left[\begin{array}{cc}
e \pi & f \\
g & h \pi
\end{array}\right]
$$

with $e, f, g, h \in \mathbb{Z}_{p^{2}}$ and $\Pi^{2}=p I_{2}$. If $p \nmid f$ then $M=\left[\begin{array}{cc}1 & 0 \\ e \pi & f\end{array}\right]$ is a unit in $C$, and $M \Pi M^{-1}=\left[\begin{array}{ll}0 & 1 \\ p & 0\end{array}\right]$. Similarly, if $p \nmid g$ there is $M \in C^{\times}$such that $M \Pi M^{-1}=\left[\begin{array}{ll}0 & p \\ 1 & 0\end{array}\right]$. If $p \mid f$ and $p \mid g$ then by an iterative procedure one constructs $M \in C^{\times}$such that $M \Pi M^{-1}=\left[\begin{array}{ll}\pi & 0 \\ 0 & \pi\end{array}\right]$. Hence, up to conjugation by units in $C$, there are three possibilities for $\left(i_{t} \otimes \mathbb{Z}_{p}\right)(\pi)$, namely

$$
\Pi_{1}=\left[\begin{array}{ll}
0 & 1 \\
p & 0
\end{array}\right] \quad \Pi_{2}=\left[\begin{array}{ll}
0 & p \\
1 & 0
\end{array}\right] \quad \Pi_{3}=\left[\begin{array}{ll}
\pi & 0 \\
0 & \pi
\end{array}\right] .
$$

Corresponding to the matrices in (4.7) are embeddings $i_{p}^{1}, i_{p}^{2}$, and $i_{p}^{3}$ of $\hat{\mathcal{O}}_{1, p}$ into $\mathbb{M}_{2}\left(\hat{\mathcal{O}}_{1, p}\right)$ such that $i_{p}^{j}(\pi)=\Pi_{j}$. If $i: \mathcal{O}_{1, N^{-}} \rightarrow \operatorname{End}(E \times E)$ is a special 
embedding then $i \otimes \mathbb{Z}_{p}$ is conjugate to exactly one of these embeddings. We say that $i$ is of type $j$ if $i \otimes \mathbb{Z}_{p}$ is conjugate to $i_{p}^{j}$. The commutant of the image of $i_{p}^{1}$ consists of matrices of the form (4.5) with $d=a$ and $c=p b$; the commutant of the image of $i_{p}^{2}$ consists of matrices of the form (4.5) with $d=a$ and $b=p c$; and the commutant of the image of $i_{p}^{3}$ consists of matrices of the form (4.5) with $a, b, c, d \in \mathbb{Z}_{p}$. It follows that for $j=1,2$ the commutant of the image of $i_{p}^{j}$ in $\mathbb{M}_{2}\left(\hat{\mathcal{O}}_{1, p}\right)$ is a local Eichler order of type $\left(1, p^{2}\right)$, while the commutant of the image of $i_{p}^{3}$ in $\mathbb{M}_{2}\left(\hat{\mathcal{O}}_{1, p}\right)$ is a local Eichler order of type $(p, 1)$. By $(3.3)$ and Remark 2.5 we may assume $\mathcal{O}_{D_{1}} \otimes \mathbb{Z}_{p} \cong \mathbb{Z}_{p^{2}}$. Since $\mathbb{Z}_{p^{2}}$ cannot be embedded into a local Eichler order of type $(p, 1)$, this implies that the third case does not occur. Therefore if $p \mid N^{-}$then $\operatorname{End}\left(A_{t}, i_{t}, Z_{t}\right) \otimes \mathbb{Z}_{p}$ is a local Eichler order of type $\left(1, p^{2}\right)$.

The following definition characterizes the set of potential intersection points in characteristic $p$.

Definition 4.1. Let $p$ be a prime.

(1) If $p \nmid N$ define $\mathcal{T}_{p}$ to be the set of isomorphism classes of triples $(A, i, Z)$ over $\overline{\mathbb{F}}_{p}$ such that $A \cong E \times E$ for some supersingular elliptic curve $E$.

(2) If $p \mid N^{-}$define $\mathcal{T}_{p}$ to be the set of isomorphism classes of triples $(A, i, Z)$ over $\overline{\mathbb{F}}_{p}$ such that $A \cong E \times E$ for some supersingular elliptic curve $E$ and $i$ is of type 1 or 2.

(3) If $p \mid N^{+}$define $\mathcal{T}_{p}$ to be the empty set.

Remark 4.2. In Proposition 5.1 we will show that for $p \nmid N^{+}$the set $\mathcal{T}_{p}$ parametrizes isomorphism classes of Eichler orders of type $\left(N^{+}, p N^{-}\right)$. Thus for each prime $p$ the set $\mathcal{T}_{p}$ is finite.

Let $p \nmid N^{+}$and let $(A, i, Z) \in \mathcal{T}_{p}$. We now use the orientation $\left\{\phi_{l}\right\}_{l \mid N}$ on $\mathcal{O}_{N^{+}, N^{-}}$to construct an orientation $\left\{\psi_{l}\right\}_{l \mid p N}$ on $\operatorname{End}(A, i, Z)$. For $l \neq p$ let $T_{l}(A)$ be the $l$-adic Tate module of $A$ and let $U_{l} \supset T_{l}(A)$ be the lattice which corresponds to the $l$-primary subgroup of $Z$. Then $U_{l}$ is free of rank 1 as a left module over $\mathcal{O}_{N^{+}, N^{-}} \otimes \mathbb{Z}_{l}$. Using Lemma 2.7 we get an isomorphism

$$
\operatorname{End}(A, i, Z) \otimes \mathbb{Z}_{l} \cong \operatorname{End}\left(U_{l}, i \otimes \mathbb{Z}_{l}\right) \cong \mathcal{O}_{N^{+}, N^{-}}^{o p} \otimes \mathbb{Z}_{l} .
$$

It follows that the orientation $\phi_{l}$ on $\mathcal{O}_{N^{+}, N^{-}} \otimes \mathbb{Z}_{l}$ induces an orientation $\psi_{l}$ on $\operatorname{End}(A, i, Z) \otimes \mathbb{Z}_{l}$. 
It remains to construct an orientation on $\operatorname{End}(A, i, Z) \otimes \mathbb{Z}_{p}$. It follows from the computations above (cf. (4.3) and (4.5)) that $\operatorname{End}(A, i, Z)$ acts on $\operatorname{Lie}(A)$ through scalar multiplication by elements of $\mathbb{F}_{p^{2}}$. If $p \nmid N$ this action gives an orientation

$$
\psi_{p}: \operatorname{End}(A, i, Z) \otimes \mathbb{Z}_{p} \longrightarrow \mathbb{F}_{p^{2}} \cong R_{1, p}
$$

If $p \mid N^{-}$we note that $\mathbb{Z}_{p^{2}}$ embeds in $\mathcal{O}_{1, N^{-}}$. Let $v \in \operatorname{Lie}(A)$ be an eigenvector for $\mathbb{Z}_{p^{2}}$ such that $\mathcal{O}_{1, N^{-}} \cdot v$ spans $\operatorname{Lie}(A)$. (It follows from (4.4) and (4.7) that $v$ exists and is uniquely determined up to scalar multiplication.) For every $\alpha \in \operatorname{End}(A, i, Z)$ there is $\beta \in \mathcal{O}_{1, N^{-}}$such that $\alpha \cdot v=i(\beta) \cdot v$. The map $\bar{\psi}_{p}: \operatorname{End}(A, i, Z) \rightarrow R_{1, p}$ given by $\bar{\psi}_{p}(\alpha)=\phi_{p}(\beta)$ is a well-defined ring homomorphism. Since $\operatorname{End}(A, i, Z) \otimes \mathbb{Z}_{p}$ is an Eichler order of type $\left(1, p^{2}\right)$, the homomorphism $\bar{\psi}_{p}$ lifts uniquely to an orientation

$$
\psi_{p}: \operatorname{End}(A, i, Z) \otimes \mathbb{Z}_{p} \longrightarrow R_{1, p^{2}} .
$$

Combining the above results we get the following proposition:

Proposition 4.3. (a) Let $t$ be a point of characteristic $p$ in the support of the intersection of $\mathcal{P}_{D_{1}}$ with $\mathcal{P}_{D_{2}}$. Then $\left(A_{t}, i_{t}, Z_{t}\right) \in \mathcal{T}_{p}$.

(b) Let $(A, i, Z) \in \mathcal{T}_{p}$ and let $\left\{\psi_{l}\right\}_{l \mid p N}$ be the orientation on $\operatorname{End}(A, i, Z)$ induced by the orientation $\left\{\phi_{l}\right\}_{l \mid N}$ on $\mathcal{O}_{N^{+}, N^{-}}$. Then $\left(\operatorname{End}(A, i, Z),\left\{\psi_{l}\right\}_{l \mid p N}\right)$ is an oriented Eichler order of type $\left(N^{+}, p N^{-}\right)$.

\section{FAMILIES OF EichleR ORDERS}

In this section we describe the relation between the endomorphism rings of elements of $\mathcal{T}_{p}$ and isomorphism classes of oriented Eichler orders of type $\left(N^{+}, p N^{-}\right)$. It follows from Proposition 3.1 that if $(A, i, Z) \in \mathcal{T}_{p}$ then $A \cong E \times E$ for any supersingular elliptic curve $E$ over $\overline{\mathbb{F}}_{p}$. As in $\S 4$ we assume that $E$ is defined over $\mathbb{F}_{p}$. We also let $F \in \Lambda=\operatorname{End}(E)$ denote the Frobenius endomorphism of $E$.

Proposition 5.1. (a) If $p \nmid N$ then the map $(A, i, Z) \mapsto\left(\operatorname{End}(A, i, Z),\left\{\psi_{l}\right\}_{l \mid p N}\right)$ gives a bijection between $\mathcal{T}_{p}$ and the set of isomorphism classes of oriented Eichler orders of type $\left(N^{+}, p N^{-}\right)$.

(b) If $p \mid N^{-}$then the map $(A, i, Z) \mapsto\left(\operatorname{End}(A, i, Z),\left\{\psi_{l}\right\}_{l \mid p N}\right)$ gives a bijection between the set of $(A, i, Z) \in \mathcal{T}_{p}$ such that $i$ has type 1 , and the set of isomorphism classes of oriented Eichler orders of type $\left(N^{+}, p N^{-}\right)$. The same statement holds for $i$ of type 2 . 
Proof: We first show that the given maps are onto. Let $\left(\mathcal{E},\left\{\mu_{l}\right\}_{l \mid p N}\right)$ be an oriented Eichler order of type $\left(N^{+}, p N^{-}\right)$. Let $i_{0}: \mathcal{O}_{1, N^{-}} \rightarrow \mathbb{M}_{2}\left(H_{p}\right)$ be an embedding. Since the commutant of $i_{0}\left(\mathcal{O}_{1, N^{-}}\right)$in $\mathbb{M}_{2}\left(H_{p}\right)$ is isomorphic to $\Delta(p)$, there is an embedding $h_{0}: \mathcal{E} \rightarrow \mathbb{M}_{2}\left(H_{p}\right)$ such that $h_{0}(\mathcal{E})$ commutes with $i_{0}\left(\mathcal{O}_{1, N^{-}}\right)$. We will show that there exists $g \in \mathbb{G L}_{2}\left(H_{p}\right)$ and $Z \subset E \times E$ such that $g i_{0}(x) g^{-1}$ is a special embedding of $\mathcal{O}_{1, N^{-}}$into $\mathbb{M}_{2}(\Lambda) \cong \operatorname{End}(E \times E)$ and $g h_{0}(y) g^{-1}$ gives an isomorphism between the oriented orders $\left(\mathcal{E},\left\{\mu_{l}\right\}_{l \mid p N}\right)$ and $\operatorname{End}\left(E \times E, g i_{0}(x) g^{-1}, Z\right)$. Let $l$ be a prime such that $l \mid N$ and $l \neq p$. Then the lattice $g^{-1}\left(T_{l}(E \times E)\right)$ must satisfy the following conditions:

(1) $g^{-1}\left(T_{l}(E \times E)\right)$ is stabilized by $i_{0}\left(\mathcal{O}_{1, N^{-}}\right)$and $h_{0}(\mathcal{E})$.

(2) The orientation on $\mathcal{E} \otimes \mathbb{Z}_{l}$ induced by $\phi_{l}$ with respect to $g^{-1}\left(T_{l}(E \times E)\right)$ is equal to $\mu_{l}$.

To identify an appropriate $g$ we will first find lattices with these properties.

Suppose $l \mid N^{+}$. Then $\mathbb{M}_{2}\left(H_{p}\right) \otimes \mathbb{Q}_{l} \cong \mathbb{M}_{4}\left(\mathbb{Q}_{l}\right)$ acts on $\mathbb{Q}_{l}^{4}$, and hence $i_{0}$ and $h_{0}$ induce an action of $\left(\mathcal{E} \otimes \mathbb{Z}_{l}\right) \otimes\left(\mathcal{O}_{1, N^{-}} \otimes \mathbb{Z}_{l}\right)$ on $\mathbb{Q}_{l}^{4}$. Using the Skolem-Noether theorem we may identify $\mathcal{E} \otimes \mathbb{Z}_{l}$ with the standard Eichler order $\hat{\mathcal{O}}_{l^{n_{l}^{+}}, 1}, \mathcal{O}_{1, N^{-}} \otimes \mathbb{Z}_{l}$ with $\mathbb{M}_{2}\left(\mathbb{Z}_{l}\right)$, and $\mathbb{Q}_{l}^{4}$ with $\mathbb{M}_{2}\left(\mathbb{Q}_{l}\right)$ in such a way that this action is isomorphic to $(A \otimes B) \cdot X=A X B^{\iota}$, where $A \in \hat{\mathcal{O}}_{l^{n_{l}^{+}, 1}}, B \in \mathbb{M}_{2}\left(\mathbb{Z}_{l}\right), X \in \mathbb{M}_{2}\left(\mathbb{Q}_{l}\right)$, and $\iota$ is the canonical involution of $\mathbb{M}_{2}\left(\mathbb{Q}_{l}\right)$. It follows that every lattice in $\mathbb{Q}_{l}^{4} \cong \mathbb{M}_{2}\left(\mathbb{Q}_{l}\right)$ which is stabilized by $\left(\mathcal{E} \otimes \mathbb{Z}_{l}\right) \otimes\left(\mathcal{O}_{1, N^{-}} \otimes \mathbb{Z}_{l}\right)$ is a $\mathbb{Q}_{l}^{\times}$-multiple of one of the lattices

$$
L_{j}=\left\{\left[\begin{array}{ll}
a & b \\
c & d
\end{array}\right] \in \mathbb{M}_{2}\left(\mathbb{Z}_{l}\right): l^{j} \mid c \text { and } l^{j} \mid d\right\}
$$

for $0 \leq j \leq n_{l}^{+}$.

We may assume further that the identification of $\mathcal{O}_{1, N^{-}} \otimes \mathbb{Z}_{l}$ with $\mathbb{M}_{2}\left(\mathbb{Z}_{l}\right)$ maps $\mathcal{O}_{N^{+}, N^{-}} \otimes \mathbb{Z}_{l}$ onto $\hat{\mathcal{O}}_{l^{n_{l}^{+}, 1}}$. Let $M_{j} \supset L_{j}$ be a lattice such that $M_{j} / L_{j}$ is cyclic of order $l^{n_{l}^{+}}$and $M_{j}$ is stabilized by both $\mathcal{E} \otimes \mathbb{Z}_{l}$ and $\mathcal{O}_{N^{+}, N^{-}} \otimes \mathbb{Z}_{l}$. (The lattice $M_{j}$ corresponds to the $l$-primary subgroup of $Z$.) Such a lattice $M_{j}$ exists if and only if $j=0$ or $j=n_{l}^{+}$, and in those cases

$$
M_{0}=\left\{\left[\begin{array}{cc}
a & l^{-n_{l}^{+} b} \\
c & d
\end{array}\right]: a, b, c, d \in \mathbb{Z}_{l}\right\}
$$


and $M_{n_{l}^{+}}=\hat{\mathcal{O}}_{l_{l}^{n_{l}^{+}, 1}}$ are uniquely determined. For $j=0, n_{l}^{+}$the commuting actions of $\mathcal{E} \otimes \mathbb{Z}_{l}$ and $\mathcal{O}_{N^{+}, N^{-}} \otimes \mathbb{Z}_{l}$ on $M_{j}$ induce isomorphisms between $\mathcal{E} \otimes \mathbb{Z}_{l}$ and $\mathcal{O}_{N^{+}, N^{-}}^{o p} \otimes \mathbb{Z}_{l}$ (cf. (4.8)). Since $\phi_{l}$ induces opposite orientations on $\mathcal{E} \otimes \mathbb{Z}_{l}$ with respect to the lattices $M_{0}$ and $M_{n_{l}^{+}}$, by choosing $j \in\left\{0, n_{l}^{+}\right\}$appropriately we get $M_{j}$ such that $\phi_{l}$ induces $\mu_{l}$ with respect to $M_{j}$. Let $\mathcal{A}_{l} \subset \mathbb{M}_{2}\left(H_{p}\right) \otimes \mathbb{Q}_{l}$ denote the stabilizer of $L_{j}$.

Suppose $l \mid N^{-}$with $l \neq p$. Then $\mathbb{M}_{2}\left(H_{p}\right) \otimes \mathbb{Q}_{l} \cong \mathbb{M}_{4}\left(\mathbb{Q}_{l}\right)$ acts on $\mathbb{Q}_{l}^{4}$, and hence $i_{0}$ and $h_{0}$ induce an action of $\left(\mathcal{E} \otimes \mathbb{Z}_{l}\right) \otimes\left(\mathcal{O}_{1, N^{-}} \otimes \mathbb{Z}_{l}\right)$ on $\mathbb{Q}_{l}^{4}$. Since $\mathcal{E} \otimes \mathbb{Z}_{l} \cong$ $\mathcal{O}_{1, N^{-}} \otimes \mathbb{Z}_{l} \cong \hat{\mathcal{O}}_{1, l}$, by the Skolem-Noether theorem this action is isomorphic to $(a \otimes b) \cdot x=a x b^{\iota}$, where $a, b \in \hat{\mathcal{O}}_{1, l}, x$ lies in the quaternion division ring $\hat{B}_{l}:=\hat{\mathcal{O}}_{1, l} \otimes \mathbb{Q}_{l}$, and $\iota$ is the canonical involution of $\hat{B}_{l}$. There are two $\mathbb{Q}_{l}^{\times}$equivalence classes of lattices in $\hat{B}_{l}$ stabilized by $\left(\mathcal{E} \otimes \mathbb{Z}_{l}\right) \otimes\left(\mathcal{O}_{1, N^{-}} \otimes \mathbb{Z}_{l}\right)$. These are represented by $\hat{\mathcal{O}}_{1, l}$ and $\pi \hat{\mathcal{O}}_{1, l}$, where $\pi$ is a uniformizer for $\hat{\mathcal{O}}_{1, l}$. The orientation $\phi_{l}$ on $\mathcal{O}_{N^{+}, N^{-}} \otimes \mathbb{Z}_{l}$ induces opposite orientations on $\mathcal{E} \otimes \mathbb{Z}_{l}$ with respect to $\hat{\mathcal{O}}_{1, l}$ and $\pi \hat{\mathcal{O}}_{1, l}$. Choose $j=0,1$ so that $\phi_{l}$ induces the orientation $\mu_{l}$ on $\mathcal{E} \otimes \mathbb{Z}_{l}$ with respect to $\pi^{j} \hat{\mathcal{O}}_{1, l}$, and let $\mathcal{A}_{l} \subset \mathbb{M}_{2}\left(H_{p}\right) \otimes \mathbb{Q}_{l}$ denote the stabilizer of $\pi^{j} \hat{\mathcal{O}}_{1, l}$.

Suppose $p \nmid N$. By the Skolem-Noether theorem we may identify $\mathbb{M}_{2}\left(H_{p}\right) \otimes \mathbb{Q}_{p}$ with $\mathbb{M}_{2}\left(\hat{B}_{p}\right)$ in such a way that $i_{0}\left(\mathcal{O}_{1, N^{-}}\right) \otimes \mathbb{Z}_{p}=\mathbb{M}_{2}\left(\mathbb{Z}_{p}\right)$ and $h(\mathcal{E}) \otimes \mathbb{Z}_{p}=\hat{\mathcal{O}}_{1, p} \cdot I_{2}$. Suppose $p \mid N^{-}$and assume without loss of generality that $i$ has type 1 . Then we may identify $\mathbb{M}_{2}\left(H_{p}\right) \otimes \mathbb{Q}_{p}$ with $\mathbb{M}_{2}\left(\hat{B}_{p}\right)$ so that

$$
\begin{aligned}
i_{0}\left(\mathcal{O}_{1, N^{-}}\right) \otimes \mathbb{Z}_{p} & =\left\{\left[\begin{array}{cc}
x & y \\
p y^{\prime} & x^{\prime}
\end{array}\right]: x, y \in \mathbb{Z}_{p^{2}}\right\}, \\
h_{0}(\mathcal{E}) \otimes \mathbb{Z}_{p} & =\left\{\left[\begin{array}{cc}
a & b \pi \\
p b \pi & a
\end{array}\right]: a, b \in \mathbb{Z}_{p^{2}}\right\}
\end{aligned}
$$

and $\phi_{p}$ induces the orientation $\mu_{p}$ on $\mathcal{E} \otimes \mathbb{Z}_{p}$. In either case we define $\mathcal{A}_{p}$ to be the subring of $\mathbb{M}_{2}\left(H_{p}\right) \otimes \mathbb{Q}_{p}$ which corresponds to $\mathbb{M}_{2}\left(\hat{\mathcal{O}}_{1, p}\right)$ under this identification.

Suppose $l \nmid p N$. Then $i_{0}\left(\mathcal{O}_{1, N^{-}}\right) \otimes \mathbb{Z}_{l} \cong h_{0}(\mathcal{E}) \otimes \mathbb{Z}_{l} \cong \mathbb{M}_{2}\left(\mathbb{Z}_{l}\right)$ and $\mathbb{M}_{2}\left(H_{p}\right) \otimes \mathbb{Q}_{l} \cong$ $\mathbb{M}_{4}\left(\mathbb{Q}_{l}\right)$. Let $\mathcal{A}_{l}$ denote the subring of $\mathbb{M}_{2}\left(H_{p}\right) \otimes \mathbb{Q}_{l}$ generated by $i_{0}\left(\mathcal{O}_{1, N^{-}}\right) \otimes \mathbb{Z}_{l}$ and $h_{0}(\mathcal{E}) \otimes \mathbb{Z}_{l}$. Since $h_{0}(\mathcal{E}) \otimes \mathbb{Q}_{l}$ is the commutant of $i_{0}\left(\mathcal{O}_{1, N^{-}}\right) \otimes \mathbb{Q}_{l}$ in $\mathbb{M}_{2}\left(H_{p}\right) \otimes \mathbb{Q}_{l}$, we get $\mathcal{A}_{l} \cong \mathbb{M}_{2}\left(\mathbb{Z}_{l}\right) \otimes \mathbb{M}_{2}\left(\mathbb{Z}_{l}\right) \cong \mathbb{M}_{4}\left(\mathbb{Z}_{l}\right)$.

Let $\mathcal{A}$ be the maximal order in $\mathbb{M}_{2}\left(H_{p}\right)$ such that $\mathcal{A} \otimes \mathbb{Z}_{l}=\mathcal{A}_{l}$ for all $l$. It follows from Theorems 21.6 and 34.9 in [Re] that the maximal orders of $\mathbb{M}_{2}\left(H_{p}\right)$ 
are all conjugate. Therefore there exists $g \in \mathbb{G L}_{2}\left(H_{p}\right)$ such that $g \mathcal{A} g^{-1}=\mathbb{M}_{2}(\Lambda)$. Let $i(x)=g i_{0}(x) g^{-1}$ and $h(y)=g h_{0}(y) g^{-1}$ for $x \in \mathcal{O}_{1, N^{-}}$and $y \in \mathcal{E}$. By the construction of $\mathcal{A}$ we see that $i\left(\mathcal{O}_{1, N^{-}}\right)$and $h(\mathcal{E})$ are contained in $\mathbb{M}_{2}(\Lambda) \cong$ $\operatorname{End}(E \times E)$. In addition, if $p \mid N^{-}$then it follows from (5.3) that $i: \mathcal{O}_{1, N^{-}} \rightarrow$ $\operatorname{End}(E \times E)$ is a special embedding of type 1. Let $Z$ be the unique cyclic subgroup of $E \times E$ of order $N^{+}$which is stabilized by both $h(\mathcal{E})$ and $i\left(\mathcal{O}_{N^{+}, N^{-}}\right)$. Then we have $\mathcal{E} \cong \operatorname{End}(E \times E, i, Z)$. Let $\left\{\psi_{l}\right\}_{l \mid p N}$ be the orientation on $\operatorname{End}(E \times E, i, Z)$ induced by $\left\{\phi_{l}\right\}_{l \mid N}$. Then by the constructions above we have $\psi_{l} \circ h=\mu_{l}$ for all $l$ such that $l \mid N$. Thus if $p \mid N$ then $h$ induces an isomorphism

$$
\left(\mathcal{E},\left\{\mu_{l}\right\}_{l \mid p N}\right) \cong\left(\operatorname{End}(E \times E, i, Z),\left\{\psi_{l}\right\}_{l \mid p N}\right)
$$

as required. Suppose $p \nmid N$. If $\psi_{p} \circ h=\mu_{p}$ then (5.5) still holds, while if $\psi_{p} \circ h \neq \mu_{p}$ then (5.5) holds after we replace $(i(x), h(x), Z)$ with $\left(F i(x) F^{-1}, F h(x) F^{-1}, F(Z)\right)$.

To prove that our maps are one-to-one we need to show that if the endomorphism rings of the triples $\left(A_{1}, i_{1}, Z_{1}\right)$ and $\left(A_{2}, i_{2}, Z_{2}\right)$ are isomorphic as oriented orders then $\left(A_{1}, i_{1}, Z_{1}\right) \cong\left(A_{2}, i_{2}, Z_{2}\right)$. We may assume that $A_{1}=A_{2}=E \times E$. Then there is an oriented Eichler order $\left(\mathcal{E},\left\{\psi_{l}\right\}_{l \mid p N}\right)$ in $\Delta(p)$ of type $\left(N^{+}, p N^{-}\right)$, and embeddings $h_{j}: \mathcal{E} \rightarrow \mathbb{M}_{2}(\Lambda)$ for $j=1,2$ which induce isomorphisms between the oriented orders $\left(\mathcal{E},\left\{\psi_{l}\right\}_{l \mid p N}\right)$ and $\operatorname{End}\left(E \times E, i_{j}, Z_{j}\right)$. We need to show there is $g \in \mathbb{G L}_{2}(\Lambda)$ such that $Z_{2}=g Z_{1}$ and $i_{2}(x)=g i_{1}(x) g^{-1}$ for all $x \in \mathcal{O}_{1, N^{-}}$.

By the Skolem-Noether theorem there is $g \in \mathbb{G L}_{2}\left(H_{p}\right)$ such that $i_{2}(x)=$ $g i_{1}(x) g^{-1}$ and $h_{2}(y)=g h_{1}(y) g^{-1}$ for all $x \in \mathcal{O}_{1, N^{-}}$and $y \in \mathcal{E}$. We may assume that $g \in \mathbb{M}_{2}(\Lambda)$, and that $|\operatorname{Nr}(g)|$ is as small as possible. We claim that $g \in \mathbb{G L}_{2}(\Lambda)$. Let $R$ be the order in $\mathbb{M}_{2}(\Lambda)$ generated by $i_{2}\left(\mathcal{O}_{1, N^{-}}\right)$and $h_{2}(\mathcal{E})$. For $l \neq p$ the lattices $T_{l}=T_{l}(E \times E)$ and $g\left(T_{l}\right)$ are stabilized by $i_{2}\left(\mathcal{O}_{1, N^{-}}\right) \otimes \mathbb{Z}_{l}=g i_{1}\left(\mathcal{O}_{1, N^{-}}\right) g^{-1} \otimes \mathbb{Z}_{l}$ and $h_{2}(\mathcal{E}) \otimes \mathbb{Z}_{l}=g h_{1}(\mathcal{E}) g^{-1} \otimes \mathbb{Z}_{l}$, and hence also by $R \otimes \mathbb{Z}_{l}$.

Suppose $l \mid N^{-}$and $l \neq p$. In the existence proof we showed that there are two $\mathbb{Q}_{l}^{\times}$-equivalence classes of lattices $L$ in $T_{l} \otimes \mathbb{Q}_{l}$ stabilized by $i_{2}\left(\mathcal{O}_{1, N^{-}}\right) \otimes \mathbb{Z}_{l}$ and $h_{2}(\mathcal{E}) \otimes \mathbb{Z}_{l}$. The orientation $\phi_{l}$ on $\mathcal{O}_{N^{+}, N^{-}} \otimes \mathbb{Z}_{l}$ induces opposite orientations on $\mathcal{E} \otimes \mathbb{Z}_{l}$ with respect to these two classes. Since $\phi_{l}$ induces the same orientation on $\mathcal{E} \otimes \mathbb{Z}_{l}$ with respect to $T_{l}$ and $g\left(T_{l}\right)$, it follows that $g\left(T_{l}\right)$ lies in the same $\mathbb{Q}_{l}^{\times}$equivalence class as $T_{l}$. Hence $g\left(T_{l}\right)=l^{k} T_{l}$ for some $k \geq 0$, so $g=l^{k} \cdot g_{0}$ for some $g_{0} \in \mathbb{M}_{2}(\Lambda)$. By the minimality of $|\operatorname{Nr}(g)|$ we get $k=0$, and hence $g\left(T_{l}\right)=T_{l}$. 
Suppose $l \mid N^{+}$and $l \neq p$. Let $U_{l} \supset T_{l}$ denote the lattice corresponding to the $l$-primary subgroup of $Z_{2}$. Then $U_{l} / T_{l}$ is cyclic of order $l^{n_{l}^{+}}$, and both $U_{l}$ and $g\left(U_{l}\right)$ are stabilized by $i_{2}\left(\mathcal{O}_{N^{+}, N^{-}}\right) \otimes \mathbb{Z}_{l}$ and $h_{2}(\mathcal{E}) \otimes \mathbb{Z}_{l}$. In the existence proof we showed that there are two $\mathbb{Q}_{l}^{\times}$-equivalence classes of pairs of lattices $M \supset L$ in $T_{l} \otimes \mathbb{Q}_{l}$ such that $M / L$ is cyclic of order $l^{n_{l}^{+}}, M$ is stabilized by $i_{2}\left(\mathcal{O}_{N^{+}, N^{-}}\right) \otimes \mathbb{Z}_{l}$ and $h_{2}(\mathcal{E}) \otimes \mathbb{Z}_{l}$, and $L$ is stabilized by $i_{2}\left(\mathcal{O}_{1, N^{-}}\right) \otimes \mathbb{Z}_{l}$ and $h_{2}(\mathcal{E}) \otimes \mathbb{Z}_{l}$. The orientation $\phi_{l}$ on $\mathcal{O}_{N^{+}, N^{-}} \otimes \mathbb{Z}_{l}$ induces opposite orientations on $\mathcal{E} \otimes \mathbb{Z}_{l}$ with respect to the two $\mathbb{Q}_{l}^{\times}$-equivalence classes of lattices $M$. Since $\phi_{l}$ induces the same orientation on $\mathcal{E} \otimes \mathbb{Z}_{l}$ with respect to $U_{l}$ and $g\left(U_{l}\right)$, it follows that $g\left(U_{l}\right)$ lies in the same $\mathbb{Q}_{l}^{\times}$-equivalence class as $U_{l}$, so we have $g\left(U_{l}\right)=l^{k} U_{l}$ for some $k \geq 0$. Since $T_{l}$ is the unique sublattice of $U_{l}$ which is stabilized by $i_{2}\left(\mathcal{O}_{1, N^{-}}\right) \otimes \mathbb{Z}_{l}$ and such that $U_{l} / T_{l}$ is cyclic, it follows that $g\left(T_{l}\right)=l^{k} T_{l}$. As above this implies $g\left(T_{l}\right)=T_{l}$.

Suppose $l \nmid p N$. Then $\mathcal{O}_{1, N^{-}} \otimes \mathbb{Z}_{l} \cong \mathcal{E} \otimes \mathbb{Z}_{l} \cong \mathbb{M}_{2}\left(\mathbb{Z}_{l}\right)$ and hence $R \otimes \mathbb{Z}_{l} \cong \mathbb{M}_{4}\left(\mathbb{Z}_{l}\right)$ stabilizes $T_{l}$ and $g\left(T_{l}\right)$. It follows that $g\left(T_{l}\right)=l^{k} T_{l}$ for some $k \geq 0$. As above this implies $g\left(T_{l}\right)=T_{l}$. Hence we have $g \in \mathbb{G L}_{2}\left(\Lambda \otimes \mathbb{Z}_{l}\right)$ for every $l \neq p$.

Suppose $p \nmid N$. Then $\mathcal{O}_{1, N^{-}} \otimes \mathbb{Z}_{p} \cong \mathbb{M}_{2}\left(\mathbb{Z}_{p}\right)$ and $\mathcal{E} \otimes \mathbb{Z}_{p} \cong \hat{\mathcal{O}}_{1, p}$, which implies $R \otimes \mathbb{Z}_{p} \cong \mathbb{M}_{2}\left(\hat{\mathcal{O}}_{1, p}\right)$. Since $\Lambda^{2} \otimes \mathbb{Z}_{p} \cong \hat{\mathcal{O}}_{1, p}^{2}$ is stabilized by $R \otimes \mathbb{Z}_{p}$, we have $R \otimes \mathbb{Z}_{p}=\mathbb{M}_{2}\left(\Lambda \otimes \mathbb{Z}_{p}\right)$. The lattice $g\left(\Lambda^{2}\right) \otimes \mathbb{Z}_{p}$ is also stabilized by $R \otimes \mathbb{Z}_{p}$, so we have $g\left(\Lambda^{2}\right) \otimes \mathbb{Z}_{p}=F^{k} \cdot\left(\Lambda^{2} \otimes \mathbb{Z}_{p}\right)$ for some $k \geq 0$. It follows from the minimality of $|\operatorname{Nr}(g)|$ that $k=0$ or $k=1$. However, if $g\left(\Lambda^{2}\right) \otimes \mathbb{Z}_{p}=F \cdot\left(\Lambda^{2} \otimes \mathbb{Z}_{p}\right)$ then $g \in F \cdot \mathbb{G L}_{2}\left(\Lambda \otimes \mathbb{Z}_{p}\right)$, and hence the orientations on $\mathcal{E} \otimes \mathbb{Z}_{p}$ induced by $h_{1}(y)$ and $h_{2}(y)=g h_{1}(y) g^{-1}$ are different, contrary to assumption. Thus $g \in \mathbb{G L}_{2}\left(\Lambda \otimes \mathbb{Z}_{p}\right)$.

Suppose $p \mid N^{-}$, and assume without loss of generality that $i_{1}$ and $i_{2}$ have type 1. It follows using (5.3) and (5.4) that we may identify $\mathbb{M}_{2}\left(\Lambda \otimes \mathbb{Z}_{p}\right)$ with $\mathbb{M}_{2}\left(\hat{\mathcal{O}}_{1, p}\right)$ in such a way that $R \otimes \mathbb{Z}_{p}$ contains the matrices $\left[\begin{array}{ll}1 & 0 \\ 0 & 0\end{array}\right]$ and $\left[\begin{array}{ll}0 & 1 \\ p & 0\end{array}\right]$. Since $g\left(\Lambda^{2}\right) \otimes \mathbb{Z}_{p}$ is an $\hat{\mathcal{O}}_{1, p}$-lattice in $H_{p}^{2} \otimes \mathbb{Q}_{p} \cong \hat{B}_{p}^{2}$ which is stabilized by $R \otimes \mathbb{Z}_{p}$, it must be $\mathbb{Q}_{p}^{\times}$-equivalent to one of the lattices $\hat{\mathcal{O}}_{1, p} \oplus \hat{\mathcal{O}}_{1, p}, \hat{\mathcal{O}}_{1, p} \oplus \pi \hat{\mathcal{O}}_{1, p}, \hat{\mathcal{O}}_{1, p} \oplus p \hat{\mathcal{O}}_{1, p}$, $\pi \hat{\mathcal{O}}_{1, p} \oplus \pi \hat{\mathcal{O}}_{1, p}, \pi \hat{\mathcal{O}}_{1, p} \oplus p \hat{\mathcal{O}}_{1, p}$, or $\pi \hat{\mathcal{O}}_{1, p} \oplus p \pi \hat{\mathcal{O}}_{1, p}$. However, since $i_{1}$ and $i_{2}$ are both special embeddings, $g\left(\Lambda^{2}\right) \otimes \mathbb{Z}_{p}$ is not $\mathbb{Q}_{p}^{\times}$-equivalent to either $\hat{\mathcal{O}}_{1, p} \oplus \pi \hat{\mathcal{O}}_{1, p}$ or $\pi \hat{\mathcal{O}}_{1, p} \oplus p \hat{\mathcal{O}}_{1, p}$. Since $i_{1}$ and $i_{2}$ are both of of type $1, g\left(\Lambda^{2}\right) \otimes \mathbb{Z}_{p}$ is not equivalent to either $\hat{\mathcal{O}}_{1, p} \oplus p \hat{\mathcal{O}}_{1, p}$ or $\pi \hat{\mathcal{O}}_{1, p} \oplus \pi \hat{\mathcal{O}}_{1, p}$. Since $\phi_{p}$ induces the same orientation on $\mathcal{E} \otimes \mathbb{Z}_{p}$ with respect to the embedding pairs $\left(i_{1}, h_{1}\right)$ and $\left(i_{2}, h_{2}\right), g\left(\Lambda^{2}\right) \otimes \mathbb{Z}_{p}$ is not equivalent to $\pi \hat{\mathcal{O}}_{1, p} \oplus p \pi \hat{\mathcal{O}}_{1, p}$. We conclude that $g\left(\Lambda^{2}\right)$ is $\mathbb{Q}_{p}^{\times}$-equivalent to 
$\hat{\mathcal{O}}_{1, p} \oplus \hat{\mathcal{O}}_{1, p}$. Hence $g\left(\Lambda^{2}\right) \otimes \mathbb{Z}_{p}=p^{k} \cdot\left(\Lambda^{2} \otimes \mathbb{Z}_{p}\right)$ for some $k \geq 0$. By the minimality of $|\operatorname{Nr}(g)|$ we get $k=0$, and hence $g \in \mathbb{G L}_{2}\left(\Lambda \otimes \mathbb{Z}_{p}\right)$.

Combining the above results we get $g \in \mathbb{G L}_{2}(\Lambda)$. Finally, it follows from the explicit computations above that for $j=1,2$ the $l$-primary subgroup of $Z_{j}$ is the unique cyclic subgroup of $E \times E$ of order $l^{n_{l}^{+}}$which is stabilized by both $h_{j}(\mathcal{E})$ and $i_{j}\left(\mathcal{O}_{N^{+}, N^{-}}\right)$. Hence $g$ maps the $l$-primary subgroup of $Z_{1}$ onto the $l$-primary subgroup of $Z_{2}$. It follows that $Z_{2}=g Z_{1}$.

Recall that $\mathcal{S}_{p}$ denotes the set of Eichler orders in $\Delta(p)$ of type $\left(N^{+}, p N^{-}\right)$. When viewed as $\mathbb{Z}$-lattices in the quadratic space $\Delta(p)$, the elements of $\mathcal{S}_{p}$ all belong to the same genus. As in $\S 3$ we let $\mathcal{G}_{p}$ be a set of representatives for the proper equivalence classes of this genus.

Proposition 5.2. (a) Every $L \in \mathcal{G}_{p}$ which represents 1 over $\mathbb{Z}$ is properly equivalent to some $\mathcal{E} \in \mathcal{S}_{p}$.

(b) The orders $\mathcal{E}, \mathcal{E}^{\prime} \in \mathcal{S}_{p}$ are properly equivalent lattices if and only if $\mathcal{E}^{\prime}=a \mathcal{E} a^{-1}$ for some $a \in \Delta(p)^{\times}$.

To facilitate the proof we recall the following well-known fact (cf. [Vi, I, Th. 3.3]):

Lemma 5.3. Let $B$ be a quaternion algebra over a field $F$ whose characteristic is not 2. Then every proper self-isometry of $B$ has the form $\phi(x)=a x b^{-1}$ for some $a, b \in B^{\times}$such that $\operatorname{Nr}(a)=\operatorname{Nr}(b)$.

Proof of Proposition 5.2: (a) Let $\mathcal{E}_{0} \subset \Delta(p)$ be an Eichler order of type $\left(N^{+}, p N^{-}\right)$ and let $l$ be a prime. Since $\mathcal{E}_{0}$ and $L$ lie in the same genus, the lattices $\mathcal{E}_{0} \otimes \mathbb{Z}_{l}$ and $L \otimes \mathbb{Z}_{l}$ are equivalent. Since the order $\mathcal{E}_{0} \otimes \mathbb{Z}_{l}$ is stabilized by the canonical involution of $\Delta(p) \otimes \mathbb{Q}_{l}, \mathcal{E}_{0} \otimes \mathbb{Z}_{l}$ and $L \otimes \mathbb{Z}_{l}$ are properly equivalent. It follows from Lemma 5.3 that there are $a_{l}, b_{l} \in\left(\Delta(p) \otimes \mathbb{Q}_{l}\right)^{\times}$such that $L \otimes \mathbb{Z}_{l}=a_{l}\left(\mathcal{E}_{0} \otimes \mathbb{Z}_{l}\right) b_{l}^{-1}$.

Let $\mathcal{E}_{L} \subset \Delta(p)$ be the right order of $L$. Then $\mathcal{E}_{L} \otimes \mathbb{Z}_{l}=b_{l}\left(\mathcal{E}_{0} \otimes \mathbb{Z}_{l}\right) b_{l}^{-1}$ for every prime $l$, so we have $\mathcal{E}_{L} \in \mathcal{S}_{p}$. Let $u \in L$ be such that $\operatorname{Nr}(u)=1$. Then $u \mathcal{E}_{L} \subset L$, and for every prime $l$ the lattices $u \mathcal{E}_{L} \otimes \mathbb{Z}_{l}$ and $L \otimes \mathbb{Z}_{l}$ are both equivalent to $\mathcal{E}_{0} \otimes \mathbb{Z}_{l}$. Since $\Delta(p) \otimes \mathbb{Z}_{l}$ is a nondegenerate quadratic space, this implies $u \mathcal{E}_{L} \otimes \mathbb{Z}_{l}=L \otimes \mathbb{Z}_{l}$. Thus $u \mathcal{E}_{L}=L$, and hence $L$ is equivalent to $\mathcal{E}_{L} \in \mathcal{S}_{p}$. Since the canonical involution $\iota$ is an improper self-isometry of $\Delta(p), L$ is properly equivalent to either $\mathcal{E}_{L}$ or $\iota\left(\mathcal{E}_{L}\right)$. 
(b) If $\mathcal{E}^{\prime}=a \mathcal{E} a^{-1}$ with $a \in \Delta(p)^{\times}$then $x \mapsto a x a^{-1}$ is a proper isometry from $\mathcal{E}$ to $\mathcal{E}^{\prime}$. Conversely, suppose $\phi: \mathcal{E} \rightarrow \mathcal{E}^{\prime}$ is a proper isometry. By Lemma 5.3 we have $\phi(x)=a x b^{-1}$ for some $a, b \in \Delta(p)^{\times}$. Clearly $\phi(1)=a b^{-1}$ is a unit in $\mathcal{E}^{\prime}$, so $a \mathcal{E} a^{-1}=\phi(\mathcal{E})\left(a b^{-1}\right)^{-1}=\mathcal{E}^{\prime}$.

\section{UNIVERSAL DEFORMATIONS}

Let $\left(A_{t}, i_{t}, Z_{t}, \beta_{t}\right)$ correspond to a point $t \in \mathcal{X}\left(\overline{\mathbb{F}}_{p}\right)$, and let $W_{p}=W\left(\overline{\mathbb{F}}_{p}\right)$ denote the ring of Witt vectors with coefficients in $\overline{\mathbb{F}}_{p}$. In this section we study the completion $\hat{\mathcal{X}}_{t}$ of $\mathcal{X} \otimes W_{p}$ at $t$. Since $\mathcal{X}$ is a fine moduli space, $\hat{\mathcal{X}}_{t}$ is a universal deformation space for $\left(A_{t}, i_{t}, Z_{t}, \beta_{t}\right)$. Let $\hat{A}_{t}$ be the formal group of $A_{t}$ and let $\hat{\imath}_{t}: \mathcal{O}_{1, N^{-}} \otimes \mathbb{Z}_{p} \rightarrow \operatorname{End}\left(\hat{A}_{t}\right)$ be the map induced by $i_{t}$. To begin we show that $\hat{\mathcal{X}}_{t}$ is a universal deformation space for the pair $\left(\hat{A}_{t}, \hat{\imath}_{t}\right)$.

Lemma 6.1. Let $p$ be a prime such that $p \nmid m N^{+}$, and let $R$ be a complete Noetherian local ring with residue field $\overline{\mathbb{F}}_{p}$. Then there are natural bijections between

(a) The set of deformations over $R$ of the 4 -tuple $\left(A_{t}, i_{t}, Z_{t}, \beta_{t}\right)$,

(b) The set of deformations over $R$ of the pair $\left(A_{t}, i_{t}\right)$, and

(c) The set of deformations over $R$ of the pair $\left(\hat{A}_{t}, \hat{\imath}_{t}\right)$.

Therefore $\hat{\mathcal{X}}_{t}$ serves as a universal deformation space for either $\left(A_{t}, i_{t}, Z_{t}, \beta_{t}\right)$, $\left(A_{t}, i_{t}\right)$, or $\left(\hat{A}_{t}, \hat{\imath}_{t}\right)$.

Proof: Since $p \nmid m N^{+}$, Hensel's Lemma [Mi, I, Th. 4.2(d)] implies that if $(A, i)$ is a deformation of $\left(A_{t}, i_{t}\right)$ defined over $R$ then $Z_{t}$ extends uniquely to a subgroup scheme $Z \subset A$ which is finite and flat of order $N^{+}$over $R$, and $\beta_{t}$ extends uniquely to a $\Gamma_{1}(m)$-structure $\beta$ on $A$. This gives a bijection between (a) and (b). The Serre-Tate lifting theorem (see the appendix to $[\mathrm{Dr}]$ ) gives a bijection between (b) and (c).

Proposition 6.2. Let $p$ be a prime such that $p \nmid m N^{+}$, and let $t \in \mathcal{X}\left(\overline{\mathbb{F}}_{p}\right)$.

(a) If $p \nmid N^{-}$then $\hat{\mathcal{X}}_{t} \cong \operatorname{Spf} W[[u]]$.

(b) If $p \mid N^{-}$and $i_{t}$ is of type 1 or 2 then $\hat{\mathcal{X}}_{t} \cong \operatorname{Spf} W[[u]]$. If $p \mid N^{-}$and $i_{t}$ is of type 3 then $\hat{\mathcal{X}}_{t} \cong \operatorname{Spf} W[[u, v]] /(u v-p)$. 
Proof: (a) In this case $\hat{A}_{t}$ has multiplication by $\mathcal{O}_{1, N^{-}} \otimes \mathbb{Z}_{p} \cong \mathbb{M}_{2}\left(\mathbb{Z}_{p}\right)$ and must therefore be isomorphic to a product $G_{0} \times G_{0}$, where $G_{0}$ is a formal group of dimension 1 and height 2 over $\overline{\mathbb{F}}_{p}$. By the same reasoning any deformation of $\hat{A}_{t}$ with multiplication by $\mathcal{O}_{1, N^{-}} \otimes \mathbb{Z}_{p}$ has the form $G \times G$, where $G$ is a deformation of $G_{0}$, and conversely any deformation $G$ of $G_{0}$ gives a unique deformation $G \times G$ of $\hat{A}_{t}$ with multiplication by $\mathcal{O}_{1, N^{-}} \otimes \mathbb{Z}_{p}$. Therefore deformations of the pair $\left(\hat{A}_{t}, \hat{\imath}_{t}\right)$ are equivalent to deformations of $G_{0}$. In [LT] it is proved that the universal deformation space of the formal group $G_{0}$ is $\operatorname{Spf} W_{p}[[u]]$. Hence by Lemma 6.1 we get $\hat{\mathcal{X}}_{t} \cong \operatorname{Spf} W_{p}[[u]]$.

(b) In this case $\hat{A}_{t}$ has multiplication by $\mathcal{O}_{1, N^{-}} \otimes \mathbb{Z}_{p} \cong \hat{\mathcal{O}}_{1, p}$. We use Drinfeld's theory in $[\mathrm{Dr}]$ to interpret the formal scheme $\hat{\mathcal{H}}_{p}$ (the " $p$-adic upper half-plane") as a moduli space for rigidified formal groups of dimension 2 and height 4 with a special action by $\hat{\mathcal{O}}_{1, p}$. Associated to the pair $\left(\hat{A}_{t}, \hat{\imath}_{t}\right)$ is an equivalence class of closed points in $\hat{\mathcal{H}}_{p}$. The formal neighborhood of any of these points is a universal deformation space for $\left(\hat{A}_{t}, \hat{\imath}_{t}\right)$, and hence also for $\left(A_{t}, i_{t}, Z_{t}, \beta_{t}\right)$.

To determine the structure of this formal neighborhood we use the explicit description of $\hat{\mathcal{H}}_{p}$ found in [Te, pp. 650-51] and [BC, I, §3]. The special fiber of $\hat{\mathcal{H}}_{p}$ is an infinite tree consisting of projective lines which meet transversely at their $\mathbb{F}_{p}$-rational points. The formal neighborhood of a closed point in $\hat{\mathcal{H}}_{p}$ takes two different forms, depending on whether or not it is a crossing point of the special fiber of $\hat{\mathcal{H}}_{p}$. To distinguish the crossing points from the other points in the special fiber of $\hat{\mathcal{H}}$ we let $\pi$ be an element of $\mathcal{O}_{1, N^{-}} \otimes \mathbb{Z}_{p}$ such that $\pi^{2}=p$. Then $\pi$ is a generator for the maximal ideal of $\mathcal{O}_{1, N^{-}} \otimes \mathbb{Z}_{p}$, and $\pi$ induces an endomorphism of $\operatorname{Lie}\left(\hat{A}_{t}\right)$ whose square is zero. If $i_{t}$ is of type 1 or 2 then $\pi$ induces a non-trivial endomorphism of $\operatorname{Lie}\left(\hat{A}_{t}\right)$. In this case $t$ is not a crossing point of the special fiber of $\hat{\mathcal{H}}_{p}$, and [Te, p. 650] gives $\hat{\mathcal{X}}_{t} \cong \operatorname{Spf} W_{p}[[u]]$. If $i_{t}$ is of type 3 then $\pi$ induces the zero map on $\operatorname{Lie}\left(\hat{A}_{t}\right)$. In this case $t$ is a crossing point of the special fiber of $\hat{\mathcal{H}}_{p}$, and by [Te, p. 650] we have $\hat{\mathcal{X}}_{t} \cong \operatorname{Spf} W_{p}[[u, v]] /(u v-p)$.

The following consequence of Proposition 6.2 is presumably well-known:

Corollary 6.3. $\mathcal{X} \otimes \mathbb{Z}\left[1 / m N^{+}\right]$is a regular scheme.

Let $R$ be a complete noetherian local ring with residue field $\overline{\mathbb{F}}_{p}$ and let $(\hat{A}, \hat{\imath})$ be a deformation of $\left(\hat{A}_{t}, \hat{\imath}_{t}\right)$ defined over $R$. The reduction map $R \rightarrow \overline{\mathbb{F}}_{p}$ induces inclusions $\operatorname{End}(\hat{A}) \subset \operatorname{End}\left(\hat{A}_{t}\right)$ and $\operatorname{End}(\hat{A}, \hat{\imath}) \subset \operatorname{End}\left(\hat{A}_{t}, \hat{\imath}_{t}\right)$. Let $(\underline{\hat{A}}, \underline{\hat{\imath}})$ be a universal deformation of $\left(\hat{A}_{t}, \hat{\imath}_{t}\right)$ defined over $\hat{\mathcal{X}}_{t}$, and let $S$ be a subset of 
$\operatorname{End}\left(\hat{A}_{t}, \hat{\imath}_{t}\right)$. We define $\hat{\mathcal{X}}_{t}(S)$ to be the largest formal subscheme of $\hat{\mathcal{X}}_{t}$ such that $S \subset \operatorname{End}\left(\underline{\hat{A}} \times_{\hat{\mathcal{X}}_{t}} \hat{\mathcal{X}}_{t}(S)\right)$. The following facts are easily verified:

Lemma 6.4. (a) $\hat{\mathcal{X}}_{t}(S)$ is closed in $\hat{\mathcal{X}}_{t}$.

(b) $\hat{\mathcal{X}}_{t}\left(S_{1} \cup S_{2}\right)=\hat{\mathcal{X}}_{t}\left(S_{1}\right) \cap \hat{\mathcal{X}}_{t}\left(S_{2}\right)$.

(c) Let $\mathbb{Z}_{p}[S]$ be the $\mathbb{Z}_{p}$-subalgebra of $\operatorname{End}\left(\hat{A}_{t}, \hat{\imath}_{t}\right)$ generated by $S$. If $S \subset S^{\prime} \subset \mathbb{Z}_{p}[S]$ then $\hat{\mathcal{X}}_{t}\left(S^{\prime}\right)=\hat{\mathcal{X}}_{t}(S)$.

Let $\gamma_{1}, \gamma_{2} \in \operatorname{End}\left(\hat{A}_{t}, \hat{\imath}_{t}\right)$. The intersection multiplicity $\alpha_{p}\left(\gamma_{1}, \gamma_{2}\right)$ of $\hat{\mathcal{X}}_{t}\left(\gamma_{1}\right)$ with $\hat{\mathcal{X}}_{t}\left(\gamma_{2}\right)$ is defined to be the $W_{p}$-length of the coordinate ring of $\hat{\mathcal{X}}_{t}\left(\gamma_{1}\right) \cap \hat{\mathcal{X}}_{t}\left(\gamma_{2}\right)=$ $\hat{\mathcal{X}}_{t}\left(\left\{\gamma_{1}, \gamma_{2}\right\}\right)$. To compute the arithmetic intersection numbers of our divisors we need to evaluate $\alpha_{p}\left(\gamma_{1}, \gamma_{2}\right)$ for certain $\gamma_{1}, \gamma_{2} \in \operatorname{End}\left(\hat{A}_{t}, \hat{\imath}_{t}\right)$.

Assume first that $p$ is not ramified in $\Delta$. Then by the proof of Proposition 6.2(a), $\hat{\mathcal{X}}_{t}$ is a universal deformation space for a formal group $G_{0}$ over $\overline{\mathbb{F}}_{p}$ of dimension 1 and height 2 , and $\operatorname{End}\left(\hat{A}_{t}, \hat{\imath}_{t}\right) \cong \operatorname{End}\left(G_{0}\right) \cong \hat{\mathcal{O}}_{1, p}$. The intersection multiplicity $\alpha_{p}\left(\gamma_{1}, \gamma_{2}\right)$ may be computed using the formulas in [GK, Prop. 5.4]. In order to state these formulas we define a quadratic form over $\mathbb{Z}_{p}$,

$$
Q(x, y, z)=\operatorname{Nr}\left(x+y \gamma_{1}+z \gamma_{2}\right),
$$

where $\mathrm{Nr}$ is the reduced norm form on $\operatorname{End}\left(\hat{A}_{t}, \hat{\imath}_{t}\right) \cong \hat{\mathcal{O}}_{1, p}$. We wish to define invariants $a_{1}, a_{2}, a_{3}$ of $Q$. If $p>2$ we diagonalize $Q$ over $\mathbb{Z}_{p}$ and define $a_{1} \leq a_{2} \leq a_{3}$ to be the $p$-adic valuations of the coefficients of the diagonal form of $Q$. If $p=2$ the definition of $a_{1}, a_{2}, a_{3}$ is more complicated and may be found in [GK, §4]. In either case we have $a_{1}=0$, so by [GK, Prop. 5.4] we get

$$
\alpha_{p}\left(\gamma_{1}, \gamma_{2}\right)=\left\{\begin{array}{lll}
\frac{a_{3}-a_{2}+1}{2} p^{a_{2} / 2}+\sum_{i=0}^{\left(a_{2}-2\right) / 2}\left(a_{2}+a_{3}-4 i\right) p^{i} & \text { if } a_{2} \equiv 0 & (\bmod 2), \\
\sum_{i=0}^{\left(a_{2}-1\right) / 2}\left(a_{2}+a_{3}-4 i\right) p^{i} & \text { if } a_{2} \equiv 1 & (\bmod 2) .
\end{array}\right.
$$

Note that the intersection multiplicity depends only on the $\mathbb{Z}_{p}$-isometry class of $Q$, and not on the particular $\gamma_{1}, \gamma_{2}$ that were used to define $Q$. Therefore we may write $\alpha_{p}(Q)=\alpha_{p}\left(\gamma_{1}, \gamma_{2}\right)$. 
Now suppose that $p \mid N^{-}$is ramified in $\Delta$, and assume that $t$ lies in the support of the intersection of $\mathcal{P}_{D_{1}}$ with $\mathcal{P}_{D_{2}}$. Then $\left(A_{t}, i_{t}, Z_{t}\right) \in \mathcal{T}_{p}$ by Proposition 4.3(a). We may assume without loss of generality that $i_{t}$ is of type 1 . Then by Proposition $4.3(\mathrm{~b})$ we see that $\operatorname{End}\left(\hat{A}_{t}, \hat{\imath}_{t}\right) \cong \operatorname{End}\left(A_{t}, i_{t}, Z_{t}\right) \otimes \mathbb{Z}_{p}$ is a local Eichler order of type $\left(1, p^{2}\right)$. Suppose that for $j=1,2$ there are embeddings of $\mathcal{O}_{D_{j}}$ into $\operatorname{End}\left(A_{t}, i_{t}, Z_{t}\right)$ with image $\mathbb{Z}\left[\gamma_{j}\right]$. By (3.3) we may assume $p \nmid D_{1}$. It follows then from Remark 2.5 that $\mathcal{O}_{D_{1}} \otimes \mathbb{Z}_{p} \cong \mathbb{Z}_{p}\left[\gamma_{1}\right] \cong \mathbb{Z}_{p^{2}}$. Let $(\hat{A}, \hat{\imath})$ be a deformation of $\left(\hat{A}_{t}, \hat{\imath}_{t}\right)$ such that $\gamma_{1} \in \operatorname{End}(\hat{A}, \hat{\imath})$. Since $i_{t}$ is of type $1, \operatorname{End}(\hat{A})$ contains a subring which is conjugate in $\operatorname{End}\left(\hat{A}_{t}\right) \cong \mathbb{M}_{2}\left(\hat{\mathcal{O}}_{1, p}\right)$ to

$$
\left\{\left[\begin{array}{cc}
a & b \\
p c & d
\end{array}\right]: a, b, c, d \in \mathbb{Z}_{p^{2}}\right\} \text {. }
$$

Therefore we are in a situation much like the case where $p$ is unramified in $\Delta$ : There is a formal group $G_{0}$ over $\overline{\mathbb{F}}_{p}$ of dimension 1 and height 2 , a map $\tau: \mathbb{Z}_{p^{2}} \rightarrow \operatorname{End}\left(G_{0}\right)$, and a deformation $G$ of $G_{0}$ such that $\hat{A} \cong G \times G$ and $\tau\left(\mathbb{Z}_{p^{2}}\right) \subset \operatorname{End}(G)$. Conversely, any deformation $G$ of $G_{0}$ such that $\tau\left(\mathbb{Z}_{p^{2}}\right) \subset$ $\operatorname{End}(G)$ gives a deformation $(\hat{A}, \hat{\imath})$ of $\left(\hat{A}_{t}, \hat{\imath}_{t}\right)$ such that $\gamma_{1} \in \operatorname{End}(\hat{A}, \hat{\imath})$. It follows that $\hat{\mathcal{X}}_{t}\left(\gamma_{1}\right)$ is isomorphic to the universal deformation space of the formal $\mathbb{Z}_{p^{2}}$ module $\left(G_{0}, \tau\right)$. It is proved in [Gr] that the universal deformation of $\left(G_{0}, \tau\right)$ is the canonical lifting $\underline{G}$ of $G_{0}$ associated to $\tau$, which is defined over $W_{p}$. Therefore we have $\hat{\mathcal{X}}_{t}\left(\gamma_{1}\right) \cong \operatorname{Spf} W_{p}$ and $\hat{\mathcal{X}}_{t}\left(\left\{\gamma_{1}, \gamma_{2}\right\}\right) \cong \operatorname{Spf}\left(W_{p} /\left(p^{k+1}\right)\right)$ for some $k \geq 0$.

To determine $k$ we use an indirect argument. Let $(\hat{A}, \hat{\imath})$ be the restriction of $(\underline{\hat{A}}, \underline{\hat{\imath}})$ to $\hat{\mathcal{X}}_{t}\left(\gamma_{1}\right) \cong \operatorname{Spf} W_{p}$, and for $n \geq 0$ let $\left(\hat{A}_{n}, \hat{\imath}_{n}\right)$ be the restriction of $(\underline{\hat{A}}, \underline{\hat{\imath}})$ to $\operatorname{Spf}\left(W_{p} /\left(p^{n+1}\right)\right)$. Then $\hat{A}_{n} \cong G_{n} \times G_{n}$, where $G_{n}=\underline{G} \otimes\left(W_{p} / p^{n+1} W_{p}\right)$. By [Gr, Prop. 3.3] we have

$$
\operatorname{End}\left(G_{n}\right)=\tau\left(\mathbb{Z}_{p^{2}}\right)+p^{n} \hat{\mathcal{O}}_{1, p}
$$

Using (4.5) we get

$$
\operatorname{End}\left(\hat{A}_{n}, \hat{\imath}_{n}\right)=\left\{\left[\begin{array}{cc}
a & b \pi \\
p b \pi & a
\end{array}\right]: a \in \tau\left(\mathbb{Z}_{p^{2}}\right), b \in p^{n} \tau\left(\mathbb{Z}_{p^{2}}\right)\right\} .
$$

It follows that $\operatorname{End}\left(\hat{A}_{n}, \hat{\imath}_{n}\right)$ is a local Eichler order of type $\left(1, p^{2 n+2}\right)$.

Recall that $k$ is the largest positive integer such that $\mathbb{Z}_{p}\left[\gamma_{1}, \gamma_{2}\right]$ is contained in $\operatorname{End}\left(\hat{A}_{k}, \hat{\imath}_{k}\right)$. Since $\mathbb{Z}_{p}\left[\gamma_{1}, \gamma_{2}\right]$ and $\operatorname{End}\left(\hat{A}_{k}, \hat{\imath}_{k}\right)$ are both local Eichler orders which contain $\mathbb{Z}_{p}\left[\gamma_{1}\right] \cong \mathbb{Z}_{p^{2}}$, this implies $\mathbb{Z}_{p}\left[\gamma_{1}, \gamma_{2}\right]=\operatorname{End}\left(\hat{A}_{k}, \hat{\imath}_{k}\right)$. It follows that the 
reduced discriminant of $\mathbb{Z}_{p}\left[\gamma_{1}, \gamma_{2}\right]$ is $p^{2 k+2}$. Let $\delta$ denote the reduced discriminant of the global order $\mathbb{Z}\left[\gamma_{1}, \gamma_{2}\right]$. Then we have $v_{p}(\delta)=2 k+2$, and hence

$$
\alpha_{p}\left(\gamma_{1}, \gamma_{2}\right)=k+1=\frac{1}{2} \cdot v_{p}(\delta) .
$$

The following lemma will be used in determining the relationship between $\hat{\mathcal{X}}_{t}\left(\sigma\left(\mathcal{O}_{D}\right)\right)$ and the divisor $\mathcal{P}_{D, \pm b}$.

Lemma 6.5. Let $G_{0}$ be a formal group of dimension 1 and height 2 over $\overline{\mathbb{F}}_{p}$ and let $\hat{\mathcal{U}}$ be a universal deformation space for $G_{0}$. Let $R_{p}$ be an order in a quadratic extension $K$ of $\mathbb{Q}_{p}$, and let $\phi: R_{p} \rightarrow \operatorname{End}\left(G_{0}\right)$ be a ring homomorphism. Then $\hat{\mathcal{U}}\left(\phi\left(R_{p}\right)\right)$ is reduced.

Proof: We have $R_{p}=\mathbb{Z}_{p}+\mathbb{Z}_{p} a$ for some $a \in R_{p}$, so by Lemma 6.4 (a) we have $\hat{\mathcal{U}}(\phi(a))=\hat{\mathcal{U}}\left(\phi\left(R_{p}\right)\right)$. The subscheme $\hat{\mathcal{U}}(\phi(a))$ of $\hat{\mathcal{U}} \cong$ Spf $W[[u]]$ is defined by an equation of the form $f(u)-u=0$, where $f(u) \in W_{p}[[u]]$ (cf. [LT, p. 58]). Let $p^{k}$ be the conductor of the order $R_{p}$. Then the Weierstrass degree of $f(u)-u$ is computed in [Ke1, Th.1.1] to be

$$
\begin{aligned}
p^{k}+2 p^{k-1}+\cdots+2 p+2 & \text { if } K / \mathbb{Q}_{p} \text { is unramified, } \\
2 p^{k}+2 p^{k-1}+\cdots+2 p+2 & \text { if } K / \mathbb{Q}_{p} \text { is ramified. }
\end{aligned}
$$

The power series $f(u)-u$ is divisible by an irreducible factor corresponding to a quasi-canonical lifting of level $l$ for each $0 \leq l \leq k$. The Weierstrass degrees of these factors are computed in [Gr, Prop. 5.3] to be

1 if $K / \mathbb{Q}_{p}$ is unramified and $l=0$,

$$
p^{l}+p^{l-1} \text { if } K / \mathbb{Q}_{p} \text { is unramified and } l \geq 1,
$$

$2 p^{l} \quad$ if $K / \mathbb{Q}_{p}$ is ramified.

Comparing Weierstrass degrees we find that $f(u)-u$ is the product of the quasicanonical lifting factors for $0 \leq l \leq k$ and a unit power series. Since the quasicanonical lifting factors are irreducible and have different degrees, the quotient $W_{p}[[u]] /(f(u)-u)$ is reduced, as claimed.

Choose $D$ and $b=\left(b_{l}\right)_{l \mid N}$ as in $\S 2$, and let $t \in \mathcal{X}\left(\overline{\mathbb{F}}_{p}\right)$ be such that $\left(A_{t}, i_{t}, Z_{t}\right) \in$ $\mathcal{T}_{p}$. We wish to consider the restriction $\hat{\mathcal{P}}_{D, \pm b}$ of the divisor $\mathcal{P}_{D, \pm b}$ to the completion $\hat{\mathcal{X}}_{t}$ of $\mathcal{X} \otimes W_{p}$ at $t$. Let $M_{p}$ denote the field of fractions of $W_{p}$ and replace $X$ with $X \otimes M_{p}$ and $P_{D, \pm b}$ with $P_{D, \pm b} \otimes M_{p}$. Then $\mathcal{P}_{D, \pm b} \otimes W_{p}$ is the closure 
of $P_{D, \pm b} \otimes M_{p}$ in $\mathcal{X} \otimes W_{p}$. For each $x$ in the support of $P_{D, \pm b} \otimes M_{p}$ define $\hat{x}$ to be the closure of $x$ in $\hat{\mathcal{X}}_{t}$. Then we have $\hat{\mathcal{P}}_{D, \pm b}=\sum(\hat{x})$, where the sum is taken over all points $x$ in the support of $P_{D, \pm b} \otimes M_{p}$ such that $t \in \bar{x}$.

Let $(\underline{A}, \underline{i}, \underline{Z})$ be a universal deformation of $\left(A_{t}, i_{t}, Z_{t}\right)$ defined over $\hat{\mathcal{X}}_{t}$, and let $\left\{\psi_{l}\right\}_{l \mid p N}$ be the orientation on $\operatorname{End}\left(A_{t}, i_{t}, Z_{t}\right)$ induced by $\left\{\phi_{l}\right\}_{l \mid N}$. Define a $b$-embedding to be a ring homomorphism $\sigma: \mathcal{O}_{D} \rightarrow \operatorname{End}\left(A_{t}, i_{t}, Z_{t}\right)$ such that $\psi_{l} \circ \sigma(\sqrt{D})=b_{l}$ for all $l \mid N$. Say that $\sigma$ is a $\pm b$-embedding if $\sigma$ is either a $b$-embedding or a $(-b)$-embedding. The relation between $\hat{\mathcal{X}}_{t}\left(\sigma\left(\mathcal{O}_{D}\right)\right)$ and $\hat{\mathcal{P}}_{D, \pm b}$ is given by the following lemma.

Lemma 6.6. (a) If $\sigma$ is a \pm -embedding then $\hat{\mathcal{X}}_{t}\left(\sigma\left(\mathcal{O}_{D}\right)\right)$ is contained in the support of $\hat{\mathcal{P}}_{D, \pm b}$.

(b) Every irreducible component of the support of $\hat{\mathcal{P}}_{D, \pm b}$ lies in $\hat{\mathcal{X}}_{t}\left(\sigma\left(\mathcal{O}_{D}\right)\right)$ for some $\pm b$-embedding $\sigma$.

(c) Let $\sigma, \sigma^{\prime}$ be $\pm b$-embeddings. Then the components in the support of $\hat{\mathcal{X}}_{t}\left(\sigma\left(\mathcal{O}_{D}\right)\right)$ and $\hat{\mathcal{X}}_{t}\left(\sigma^{\prime}\left(\mathcal{O}_{D}\right)\right)$ are all different unless $\sigma\left(\mathcal{O}_{D}\right)=\sigma^{\prime}\left(\mathcal{O}_{D}\right)$.

Proof: (a) Suppose $p \nmid N^{-}$. In the proof of Proposition 6.2 (a) we showed that $\hat{\mathcal{X}}_{t}$ is a universal deformation space for a formal group $G_{0}$ of dimension 1 and height 2. The map $\sigma$ induces an embedding of $\mathcal{O}_{D} \otimes \mathbb{Z}_{p}$ into $\operatorname{End}\left(G_{0}\right)$, and $\hat{\mathcal{X}}_{t}\left(\sigma\left(\mathcal{O}_{D}\right)\right)$ is defined by the requirement that the image of this embedding should lift. It follows from Lemma 6.5 that $\hat{\mathcal{X}}_{t}\left(\sigma\left(\mathcal{O}_{D}\right)\right)$ is reduced. Since the support of $\hat{\mathcal{P}}_{D, \pm b}$ contains the closure of the characteristic 0 fiber of $\hat{\mathcal{X}}_{t}\left(\sigma\left(\mathcal{O}_{D}\right)\right)$, it suffices to show that the generic fibers of the irreducible components of $\hat{\mathcal{X}}_{t}\left(\sigma\left(\mathcal{O}_{D}\right)\right)$ all have characteristic 0, i.e., $\hat{\mathcal{X}}_{t}\left(\sigma\left(\mathcal{O}_{D}\right)\right)$ has no vertical components. But this follows from the fact that the reduction $(\bmod p)$ of a universal deformation of $G_{0}$ has endomorphism ring $\mathbb{Z}_{p}$ (cf. [Ke1, Th. 1.1]).

Suppose $p \mid N^{-}$, and assume without loss of generality that $i_{t}$ has type 1 . It follows from assumption (3.2) and Remark 2.5 that $\mathcal{O}_{D} \otimes \mathbb{Z}_{p}$ is the ring of integers in a quadratic extension of $\mathbb{Q}_{p}$. Let $(\hat{A}, \hat{\imath})$ be a deformation of $\left(\hat{A}_{t}, \hat{\imath}_{t}\right)$ such that $\sigma\left(\mathcal{O}_{D}\right) \otimes \mathbb{Z}_{p} \subset \operatorname{End}(\hat{A}, \hat{\imath})$. Since $\operatorname{End}(\hat{A}, \hat{\imath})$ is contained in $\operatorname{End}\left(\hat{A}_{t}, \hat{\imath}_{t}\right)$, which is a local Eichler order of type $\left(1, p^{2}\right)$, we must have $\mathcal{O}_{D} \otimes \mathbb{Z}_{p} \cong \mathbb{Z}_{p^{2}}$. By the Skolem-Noether theorem there exists an isomorphism $\hat{A}_{t} \cong G_{0} \times G_{0}$ which 
identifies $i_{t}\left(\mathcal{O}_{1, N^{-}}\right) \otimes \mathbb{Z}_{p}$ with

$$
\left\{\left[\begin{array}{cc}
x & y \\
p y^{\prime} & x^{\prime}
\end{array}\right]: x, y \in \mathbb{Z}_{p^{2}}\right\}
$$

and $\sigma\left(\mathcal{O}_{D}\right) \otimes \mathbb{Z}_{p}$ with $\mathbb{Z}_{p^{2}} \cdot I_{2}$. It follows that $\hat{A}$ has multiplication by the ring

$$
\left\{\left[\begin{array}{cc}
a & b \\
p c & d
\end{array}\right]: a, b, c, d \in \mathbb{Z}_{p^{2}}\right\}
$$

generated by $\mathbb{Z}_{p^{2}} \cdot I_{2}$ and (6.9). Therefore $\hat{A} \cong G \times G$, where $G$ is a deformation of $G_{0}$. Furthermore, $\sigma$ induces an embedding $\tau: \mathcal{O}_{D} \otimes \mathbb{Z}_{p} \rightarrow \operatorname{End}\left(G_{0}\right)$ whose image is contained in $\operatorname{End}(G)$. Conversely, any deformation $G$ of $G_{0}$ such that $\tau\left(\mathcal{O}_{D} \otimes \mathbb{Z}_{p}\right) \subset \operatorname{End}(G)$ gives a deformation $(\hat{A}, \hat{\imath})$ of $\left(\hat{A}_{t}, \hat{\imath}_{t}\right)$ such that $\sigma\left(\mathcal{O}_{D}\right) \otimes \mathbb{Z}_{p} \subset \operatorname{End}(\hat{A}, \hat{\imath})$. The maximal deformation $G$ of $G_{0}$ such that $\tau\left(\mathcal{O}_{D} \otimes \mathbb{Z}_{p}\right) \subset \operatorname{End}(G)$ is the canonical lifting of $G_{0}$ associated to $\tau$. By [Gr, Prop. 2.1] the canonical lifting is defined over an integral domain of characteristic 0 . As above this implies that $\hat{\mathcal{X}}_{t}\left(\sigma\left(\mathcal{O}_{D}\right)\right)$ is contained in the support of $\hat{\mathcal{P}}_{D, \pm b}$.

(b) Let $\hat{y}$ be an irreducible component of $\hat{\mathcal{P}}_{D, \pm b}$, and let $\left(A_{y}, i_{y}, Z_{y}\right)$ be the triple corresponding to $y$. Then there are two embeddings $\rho_{y}, \bar{\rho}_{y}: \mathcal{O}_{D} \rightarrow$ $\operatorname{End}\left(A_{y}, i_{y}, Z_{y}\right)$, where $\bar{\rho}_{y}$ is $\rho_{y}$ composed with complex conjugation on $\mathcal{O}_{D}$. Let

$$
j: \operatorname{End}\left(A_{y}, i_{y}, Z_{y}\right) \longrightarrow \operatorname{End}\left(A_{t}, i_{t}, Z_{t}\right)
$$

be the natural embedding and define $\sigma=j \circ \rho_{y}$. Then $y$ is contained in $\mathcal{X}\left(\sigma\left(\mathcal{O}_{D}\right)\right)$, and hence $\hat{y}$ is contained in $\hat{\mathcal{X}}_{t}\left(\sigma\left(\mathcal{O}_{D}\right)\right)$. We need to show that $\sigma: \mathcal{O}_{D} \rightarrow \operatorname{End}\left(A_{t}, i_{t}, Z_{t}\right)$ is a $\pm b$-embedding.

Suppose $l \mid N$ with $l \neq p$. Let $T_{l}\left(A_{y}\right), T_{l}\left(A_{t}\right)$ be the $l$-adic Tate modules of $A_{y}, A_{t}$, and let $U_{l}^{y} \supset T_{l}\left(A_{y}\right), U_{l}^{t} \supset T_{l}\left(A_{t}\right)$ be the lattices which correspond to the $l$-primary subgroups of $Z_{y}, Z_{t}$. There is a natural $\left(\mathcal{O}_{N^{+}, N^{-}} \otimes \mathbb{Z}_{l}\right)$-linear isomorphism $\nu: U_{l}^{y} \rightarrow U_{l}^{t}$ such that $\nu\left(T_{l}\left(A_{y}\right)\right)=T_{l}\left(A_{t}\right)$. For $\gamma \in \operatorname{End}\left(A_{y}, i_{y}, Z_{y}\right)$ let $\tilde{\gamma}$ denote the endomorphism of $U_{l}^{y}$ induced by $\gamma$, and let $\widetilde{j(\gamma)}$ denote the endomorphism of $U_{l}^{t}$ induced by $j(\gamma)$. Then we have $\nu \circ \tilde{\gamma}=\widetilde{j(\gamma)} \circ \nu$. It now follows from the definitions of $\omega_{l}^{y}$ and $\psi_{l}$ given in $\S 2$ and $\S 4$ that $\psi_{l}(j(\gamma))=\omega_{l}^{y}(\gamma)$. Hence

$$
\psi_{l} \circ \sigma=\psi_{l} \circ j \circ \rho_{y}=\omega_{l}^{y} \circ \rho_{y} .
$$


Suppose $p \mid N^{-}$. As in the proof of (a) we see that $p$ is inert in $K$. Hence by Proposition 2.1, $T_{p}\left(A_{y}\right) / p T_{p}\left(A_{y}\right)$ is a vector space of dimension 2 over $\mathcal{R} / p \mathcal{R} \cong$ $\mathbb{F}_{p^{2}}$. Furthermore, the representation of $\operatorname{End}\left(A_{y}\right)$ on $T_{p}\left(A_{y}\right) / p T_{p}\left(A_{y}\right)$ is isomorphic to the representation of $\operatorname{End}\left(A_{y}\right)$ on $\operatorname{Lie}\left(A_{t}\right)$ induced by $j$. Therefore by the constructions of $\psi_{p}$ and $\omega_{p}^{y}$ we get $\psi_{p} \circ j \equiv \omega_{p}^{y}(\bmod p)$. It follows that

$$
\psi_{p} \circ \sigma \equiv \psi_{p} \circ j \circ \rho_{y} \equiv \omega_{p}^{y} \circ \rho_{y} \quad(\bmod p) .
$$

Since $y$ lies in the support of $P_{D, \pm b}$, it follows from (6.12) and (6.13) that $\sigma$ is a $\pm b$-embedding.

(c) Suppose $\hat{\mathcal{X}}_{t}\left(\sigma\left(\mathcal{O}_{D}\right)\right)$ and $\hat{\mathcal{X}}_{t}\left(\sigma^{\prime}\left(\mathcal{O}_{D}\right)\right)$ have a component in common. Then by (a) this component is contained in the support of $\hat{\mathcal{P}}_{D, \pm b}$. Let $y$ be the generic point of this component. Then $y$ has characteristic 0, so by Proposition 2.1, $\operatorname{End}\left(A_{y}, i_{y}, Z_{y}\right)$ is an order in $\mathbb{Q}(\sqrt{D})$. Since $\sigma\left(\mathcal{O}_{D}\right)$ and $\sigma^{\prime}\left(\mathcal{O}_{D}\right)$ both lie in $\operatorname{End}\left(A_{y}, i_{y}, Z_{y}\right)$, we must have $\sigma\left(\mathcal{O}_{D}\right)=\sigma^{\prime}\left(\mathcal{O}_{D}\right)$.

Remark 6.7. Suppose $p$ is ramified in $\Delta$ and $p$ divides the conductor $c$ of $\mathcal{O}_{D}$. Then $\hat{\mathcal{X}}_{t}\left(\sigma\left(\mathcal{O}_{D}\right)\right)$ contains the subscheme of $\hat{\mathcal{X}}_{t}$ defined by the ideal $(p)$. Therefore (a) and (c) of Lemma 6.6 are false if $p \mid c$. (However, (b) holds even if $p \mid c$.)

Proposition 6.8. We have

$$
\begin{array}{ll}
\hat{\mathcal{P}}_{D, \pm b}=\frac{1}{2} \cdot \sum \mathcal{X}_{t}\left(\sigma\left(\mathcal{O}_{D}\right)\right) & \text { if } b \neq-b, \\
\hat{\mathcal{P}}_{D, \pm b}=\sum \mathcal{X}_{t}\left(\sigma\left(\mathcal{O}_{D}\right)\right) & \text { if } b=-b,
\end{array}
$$

where the sums are taken over all $\pm b$-embeddings $\sigma: \mathcal{O}_{D} \rightarrow \operatorname{End}\left(A_{t}, i_{t}, Z_{t}\right)$.

Proof: This follows from Lemma 6.6. The factor $\frac{1}{2}$ arises in the first formula because $\sigma$ has the same image as $\bar{\sigma}$, where $\bar{\sigma}$ is $\sigma$ composed with complex conjugation. The factor $\frac{1}{2}$ is missing in the second formula because $P_{D, \pm b}=2 P_{D, b}$ when $b=-b$.

\section{Completion of the Proofs}

In this section we use the results proved in $\S 4-\S 6$ to compute $\left\langle\mathcal{P}_{D_{1}, \pm b_{1}} \cdot \mathcal{P}_{D_{2}, \pm b_{2}}\right\rangle_{\mathcal{X}}$ and $\left\langle\mathcal{P}_{D_{1}} \cdot \mathcal{P}_{D_{2}}\right\rangle_{\mathcal{X}}$. We first derive a preliminary formula for $\left\langle\mathcal{P}_{D_{1}, \pm b_{1}} \cdot \mathcal{P}_{D_{2}, \pm b_{2}}\right\rangle_{\mathcal{X}}$. 
It follows from (4.1) that there are integers $\left(\mathcal{P}_{D_{1}, \pm b_{1}} \cdot \mathcal{P}_{D_{2}, \pm b_{2}}\right)_{p}$ such that

$$
\left\langle\mathcal{P}_{D_{1}, \pm b_{1}} \cdot \mathcal{P}_{D_{2}, \pm b_{2}}\right\rangle_{\mathcal{X}}=\sum_{p<\infty}\left(\mathcal{P}_{D_{1}, \pm b_{1}} \cdot \mathcal{P}_{D_{2}, \pm b_{2}}\right)_{p} \cdot \log p
$$

The quantity $\left(\mathcal{P}_{D_{1}, \pm b_{1}} \cdot \mathcal{P}_{D_{2}, \pm b_{2}}\right)_{p}$ can be interpreted as an intersection multiplicity on $\mathcal{X} \otimes W_{p}$. Let $\mathcal{I}_{p}$ denote the set of points $t \in \mathcal{X}\left(\overline{\mathbb{F}}_{p}\right)$ such that $\left(A_{t}, i_{t}, Z_{t}\right) \in \mathcal{T}_{p}$. Then the support of the intersection of $\mathcal{P}_{D_{1}, \pm b_{1}} \otimes W_{p}$ with $\mathcal{P}_{D_{2}, \pm b_{2}} \otimes W_{p}$ on $\mathcal{X} \otimes W_{p}$ is contained in $\mathcal{I}_{p}$. Therefore we have

$$
\left(\mathcal{P}_{D_{1}, \pm b_{1}} \cdot \mathcal{P}_{D_{2}, \pm b_{2}}\right)_{p}=\sum_{t \in \mathcal{I}_{p}}\left(\mathcal{P}_{D_{1}, \pm b_{1}} \cdot \mathcal{P}_{D_{2}, \pm b_{2}}\right)_{t} .
$$

Given embeddings $\sigma_{1}, \sigma_{2}$ of $\mathcal{O}_{D_{1}}, \mathcal{O}_{D_{2}}$ into $\operatorname{End}\left(A_{t}, i_{t}, Z_{t}\right)$, set $\epsilon_{j}=\sigma_{j}\left(\sqrt{D_{j}}\right)$ and $\gamma_{j}=\left(D_{j}+\epsilon_{j}\right) / 2$ for $j=1,2$. Then $\gamma_{j} \in \operatorname{End}\left(A_{t}, i_{t}, Z_{t}\right)$ and $\sigma_{j}\left(\mathcal{O}_{D_{j}}\right)=\mathbb{Z}_{p}\left[\gamma_{j}\right]$. Hence by Lemma 6.4(c) and Proposition 6.8 we have

$$
\begin{aligned}
& \left(\mathcal{P}_{D_{1}, \pm b_{1}} \cdot \mathcal{P}_{D_{2}, \pm b_{2}}\right)_{t}=\frac{1}{4} \cdot \sum_{\sigma_{1}, \sigma_{2}} \alpha_{p}\left(\gamma_{1}, \gamma_{2}\right) \text { if } b_{1} \neq-b_{1} \text { and } b_{2} \neq-b_{2}, \\
& \left(\mathcal{P}_{D_{1}, \pm b_{1}} \cdot \mathcal{P}_{D_{2}, \pm b_{2}}\right)_{t}=\frac{1}{2} \cdot \sum_{\sigma_{1}, \sigma_{2}} \alpha_{p}\left(\gamma_{1}, \gamma_{2}\right) \text { if } b_{1}=-b_{1} \text { or } b_{2}=-b_{2},
\end{aligned}
$$

where the sums are taken over all pairs $\left(\sigma_{1}, \sigma_{2}\right)$ such that $\sigma_{j}$ is a $\pm b_{j}$-embedding. Note that since $N>1$, assumption (3.3) implies that we can't have both $b_{1}=-b_{1}$ and $b_{2}=-b_{2}$.

Let $t \in \mathcal{I}_{p}$. Then by Proposition $4.3 \operatorname{End}\left(A_{t}, i_{t}, Z_{t}\right)$ is an Eichler order of type $\left(N^{+}, p N^{-}\right)$in the quaternion algebra $\Delta(p)$ over $\mathbb{Q}$. Therefore $\operatorname{End}\left(A_{t}, i_{t}, Z_{t}\right)$ has reduced discriminant $N^{+} \cdot p N^{-}=p N$. Let $n=\frac{1}{2} \cdot \operatorname{Tr}\left(\epsilon_{1} \epsilon_{2}\right)=2 \operatorname{Tr}\left(\gamma_{1} \gamma_{2}\right)-D_{1} D_{2}$, where $\operatorname{Tr}$ is the reduced trace from $\Delta(p)$ to $\mathbb{Q}$. Since $\sigma_{j}$ is a $\pm b_{j}$-embedding we have $n \equiv \pm h(\bmod 2 N)$, where $h$ is determined by (3.9) and (3.10). Since $n \equiv D_{1} D_{2}(\bmod 2)$, the ring $S_{n}$ from $\S 1$ is defined. In fact $S_{n} \cong \mathbb{Z}\left[\gamma_{1}, \gamma_{2}\right]$ is isomorphic to a suborder of $\operatorname{End}\left(A_{t}, i_{t}, Z_{t}\right)$, and hence $B_{n} \cong \Delta(p)$ and $p N$ divides the reduced discriminant $\delta_{n}=\left(n^{2}-D_{1} D_{2}\right) / 4$ of $S_{n}$. Since $\Delta(p)$ is ramified at $\infty$ we have $\delta_{n}<0$, and hence $n^{2}<D_{1} D_{2}$. It follows that $p \leq\left|\delta_{n}\right| / N \leq D_{1} D_{2} / 4 N$, so by assumption (2.4) we have $p \nmid m$.

The ternary quadratic form $\operatorname{Nr}\left(x+y \gamma_{1}+z \gamma_{2}\right)$ over $\mathbb{Z}$ is equal to the form $Q_{n}(x, y, z)$ defined in (1.5). In $\S 6$ we saw that the intersection number $\alpha_{p}\left(\gamma_{1}, \gamma_{2}\right)$ depends only on the $\mathbb{Z}_{p}$-isometry class of $Q_{n}$ and not on $\gamma_{1}, \gamma_{2}$. Therefore we may write $\alpha_{p}\left(Q_{n}\right)=\alpha_{p}\left(\gamma_{1}, \gamma_{2}\right)$. Let $r_{t}\left(n, \pm b_{1}, \pm b_{2}\right)$ denote the number of pairs $\left(\sigma_{1}, \sigma_{2}\right)$ 
of $\pm b_{j}$-embeddings such that $\frac{1}{2} \cdot \operatorname{Tr}\left(\epsilon_{1} \epsilon_{2}\right)=n$, let $r_{t}\left(n, b_{1}, b_{2}\right)$ denote the number of such pairs which are $\left(b_{1}, b_{2}\right)$-embeddings, and let $r_{t}\left(n, \pm\left(b_{1}, b_{2}\right)\right)$ denote the number of such pairs which are either $\left(b_{1}, b_{2}\right)$-embeddings or $\left(-b_{1},-b_{2}\right)$-embeddings. If $b_{1} \neq-b_{1}$ and $b_{2} \neq-b_{2}$ then by (7.2) and (7.3) we get

$$
\begin{aligned}
\left(\mathcal{P}_{D_{1}, \pm b_{1}} \cdot \mathcal{P}_{D_{2}, \pm b_{2}}\right)_{p} & =\sum_{t \in \mathcal{I}_{p}}\left(\frac{1}{4} \cdot \sum_{\sigma_{1}, \sigma_{2}} \alpha_{p}\left(\gamma_{1}, \gamma_{2}\right)\right) \\
& =\frac{1}{4} \cdot \sum_{\substack{n^{2}<D_{1} D_{2} \\
n \equiv \pm h(2 N)}}\left(\sum_{t \in \mathcal{I}_{p}} r_{t}\left(n, \pm b_{1}, \pm b_{2}\right)\right) \cdot \alpha_{p}\left(Q_{n}\right) .
\end{aligned}
$$

Since $r_{t}\left(n, b_{1}, b_{2}\right)=r_{t}\left(-n,-b_{1}, b_{2}\right)$ and $\alpha_{p}\left(Q_{n}\right)=\alpha_{p}\left(Q_{-n}\right)$ this implies

$$
\left(\mathcal{P}_{D_{1}, \pm b_{1}} \cdot \mathcal{P}_{D_{2}, \pm b_{2}}\right)_{p}=\frac{1}{2} \cdot \sum_{\substack{n^{2}<D_{1} D_{2} \\ n \equiv h(2 N)}}\left(\sum_{t \in \mathcal{I}_{p}} r_{t}\left(n, \pm\left(b_{1}, b_{2}\right)\right)\right) \cdot \alpha_{p}\left(Q_{n}\right) .
$$

If $b_{1}=-b_{1}$ and $b_{2} \neq-b_{2}$ then by (7.2) and (7.4) we get

$$
\left(\mathcal{P}_{D_{1}, \pm b_{1}} \cdot \mathcal{P}_{D_{2}, \pm b_{2}}\right)_{p}=\frac{1}{2} \cdot \sum_{\substack{n^{2}<D_{1} D_{2} \\ n \equiv \pm h(2 N)}}\left(\sum_{t \in \mathcal{I}_{p}} r_{t}\left(n, \pm b_{1}, \pm b_{2}\right)\right) \cdot \alpha_{p}\left(Q_{n}\right)
$$

Since $\left(-b_{1}, b_{2}\right)=\left(b_{1}, b_{2}\right),\left(b_{1},-b_{2}\right)=-\left(b_{1}, b_{2}\right)$, and $h \equiv-h(\bmod 2 N)$, this equation is equivalent to (7.7). A similar calculation shows that (7.7) is also valid if $b_{2}=-b_{2}$ and $b_{1} \neq-b_{1}$. Since we can't have both $b_{1}=-b_{1}$ and $b_{2}=-b_{2}$, (7.7) is valid in all cases.

We wish to interpret the inner sum of (7.7) as counting embeddings of the ring $S_{n}$ into Eichler orders. Let $\mathcal{E} \subset \Delta(p)$ be an Eichler order of type $\left(N^{+}, p N^{-}\right)$, and let

$$
N(\mathcal{E})=\left\{\beta \in \Delta(p)^{\times}: \beta \mathcal{E} \beta^{-1}=\mathcal{E}\right\} .
$$

Then $N(\mathcal{E}) / \mathbb{Q}^{\times} \mathcal{E}^{\times}$acts freely on the set of orientations on $\mathcal{E}$; the orbits of this action are the isomorphism classes of orientations on $\mathcal{E}$. Let $v_{\mathcal{E}}=\left|N(\mathcal{E}) / \mathbb{Q}^{\times} \mathcal{E}^{\times}\right|$ and recall that $r$ is the number of distinct primes dividing $N$. Thus if $p \nmid N^{-}$ then $\mathcal{E}$ has $2^{r+1}$ orientations, and hence $2^{r+1} / v_{\mathcal{E}}$ isomorphism classes of orientations, while if $p \mid N^{-}$then $\mathcal{E}$ has $2^{r}$ orientations, and hence $2^{r} / v_{\mathcal{E}}$ isomorphism classes of orientations. By Proposition 4.3, $\operatorname{End}\left(A_{t}, i_{t}, Z_{t}\right)$ is an Eichler order 
of type $\left(N^{+}, p N^{-}\right)$for each $t \in \mathcal{I}_{p}$. Let $\mathcal{I}_{\mathcal{E}}$ denote the set of $t \in \mathcal{I}_{p}$ such that $\operatorname{End}\left(A_{t}, i_{t}, Z_{t}\right) \cong \mathcal{E}$, and let $\mathcal{C}_{p}$ be a set of representatives for the isomorphism classes of Eichler orders of type $\left(N^{+}, p N^{-}\right)$. Then

$$
\sum_{t \in \mathcal{I}_{p}} r_{t}\left(n, \pm\left(b_{1}, b_{2}\right)\right)=\sum_{\mathcal{E} \in \mathcal{C}_{p}} \sum_{t \in \mathcal{I}_{\mathcal{E}}} r_{t}\left(n, \pm\left(b_{1}, b_{2}\right)\right) \text {. }
$$

Given an orientation $\left\{\mu_{l}\right\}_{l \mid p N}$ on the Eichler order $\mathcal{E}$, say that the homomorphism $\tau: S_{n} \rightarrow \mathcal{E}$ is a $\left(b_{1}, b_{2}\right)$-embedding if $\mu_{l} \circ \tau\left(e_{j}\right) \equiv\left(b_{j}\right)_{l}\left(\bmod l^{a_{l}}\right)$ for $j=1,2$ and all $l \mid N$, where $a_{l}=v_{l}(2 N)$. Say that $\tau$ is a $\pm\left(b_{1}, b_{2}\right)$-embedding if $\tau$ is a $\left(b_{1}, b_{2}\right)$-embedding or a $\left(-b_{1},-b_{2}\right)$-embedding. Let

$$
\mathcal{I}_{\mathcal{E}}=\left\{(A, i, Z) \in \mathcal{T}_{p}: \operatorname{End}(A, i, Z) \cong \mathcal{E}\right\}
$$

and let $\tilde{\mathcal{I}}_{\mathcal{E}}$ be a subset of $\mathcal{I}_{\mathcal{E}}$ such that each $(A, i, Z) \in \mathcal{T}_{\mathcal{E}}$ is isomorphic to $\left(A_{t}, i_{t}, Z_{t}\right)$ for exactly one $t \in \tilde{\mathcal{I}}_{\mathcal{E}}$.

Let $r_{\mathcal{E}}(n)$ denote the number of embeddings of $S_{n}$ into $\mathcal{E}$. Suppose $p \nmid N$. Then each homomorphism $\tau: S_{n} \rightarrow \mathcal{E}$ is a $\pm\left(b_{1}, b_{2}\right)$-embedding for four different orientations on $\mathcal{E}$. Hence the set

$$
\Upsilon_{n}=\left\{\left(\tau: S_{n} \rightarrow \mathcal{E},\left\{\mu_{l}\right\}_{l \mid p N}\right): \tau \text { is a } \pm\left(b_{1}, b_{2}\right) \text {-embedding w.r.t. }\left\{\mu_{l}\right\}_{l \mid p N}\right\}
$$

has cardinality $4 r_{\mathcal{E}}(n)$. It follows from Proposition 5.1(a) that the isomorphism classes of orientations on $\mathcal{E}$ correspond to elements $t \in \tilde{\mathcal{I}}_{\mathcal{E}}$. This allows us to count the elements of $\Upsilon_{n}$ in a different manner, and gives the formula

$$
4 r_{\mathcal{E}}(n)=v_{\mathcal{E}} \cdot \sum_{t \in \tilde{\mathcal{I}}_{\mathcal{E}}} r_{t}\left(n, \pm\left(b_{1}, b_{2}\right)\right) .
$$

Suppose $p \mid N^{-}$. Then each homomorphism $\tau: S_{n} \rightarrow \mathcal{E}$ is a $\pm\left(b_{1}, b_{2}\right)$-embedding for two orientations on $\mathcal{E}$, and by Proposition 5.1(b) each isomorphism class of orientations on $\mathcal{E}$ is represented by two different $t \in \tilde{\mathcal{I}}_{\mathcal{E}}$. Hence (7.13) is valid in this case as well.

Let $(A, i, Z) \in \mathcal{T}_{\mathcal{E}}$. Then the group $\operatorname{Aut}(A, i, Z) \cong \mathcal{E}^{\times}$acts freely on the set of level- $m$ structures $\beta$ on $(A, i)$. By Remark 3.4 the pair $(A, i)$ admits $2 \eta(m)$ different level- $m$ structures. Hence there are $2 \eta(m) / 2 u_{\mathcal{E}}$ isomorphism classes of 4 -tuples $(A, i, Z, \beta)$, where $u_{\mathcal{E}}=\#\left(\mathcal{E}^{\times} / \pm 1\right)$. It follows that

$$
\sum_{t \in \mathcal{I}_{\mathcal{E}}} r_{t}\left(n, \pm\left(b_{1}, b_{2}\right)\right)=\frac{\eta(m)}{u_{\mathcal{E}}} \cdot \sum_{t \in \tilde{\mathcal{I}}_{\mathcal{E}}} r_{t}\left(n, \pm\left(b_{1}, b_{2}\right)\right) .
$$


Combining this formula with (7.13) we get

$$
\sum_{t \in \mathcal{I}_{\mathcal{E}}} r_{t}\left(n, \pm\left(b_{1}, b_{2}\right)\right)=\frac{4 \eta(m) r_{\mathcal{E}}(n)}{u_{\mathcal{E}} v_{\mathcal{E}}}
$$

It follows from (7.10) and (7.15) that

$$
\sum_{t \in \mathcal{I}_{p}} r_{t}\left(n, \pm\left(b_{1}, b_{2}\right)\right)=4 \eta(m) \cdot \sum_{\mathcal{E} \in \mathcal{C}_{p}} \frac{r_{\mathcal{E}}(n)}{u_{\mathcal{E}} v_{\mathcal{E}}}
$$

By combining this formula with (7.7) and (7.1) we get the following formula for the arithmetic intersection number:

$$
\left\langle\mathcal{P}_{D_{1}, \pm b_{1}} \cdot \mathcal{P}_{D_{2}, \pm b_{2}}\right\rangle_{\mathcal{X}}=2 \eta(m) \cdot \sum_{p<\infty}\left(\sum_{\substack{n^{2}<D_{1} D_{2} \\ n \equiv h(2 N)}}\left(\sum_{\mathcal{E} \in \mathcal{C}_{p}} \frac{r_{\mathcal{E}}(n)}{u_{\mathcal{E}} v_{\mathcal{E}}}\right) \cdot \alpha_{p}\left(Q_{n}\right)\right) \cdot \log p .
$$

To prove Theorem 3.2 we will evaluate the inner sum of (7.17) in terms of representation numbers of quadratic forms. To prove Theorem 3.6 we will evaluate the inner sum of (7.17) by counting Eichler orders.

Proof of Theorem 3.2: Recall that $r$ denotes the number of distinct prime divisors of $N$. Choose $n$ such that $n^{2} \equiv D_{1} D_{2}(\bmod 4 N)$. By assumption $(3.3)$ there are $2^{r}$ pairs $\left(b_{1}, b_{2}\right)$ such that $n \equiv h\left(b_{1}, b_{2}\right)(\bmod 2 N)$. If $r_{\mathcal{E}}(n) \neq 0$ then $p N$ divides the reduced discriminant $\delta_{n}=\left(n^{2}-D_{1} D_{2}\right) / 4$ of $S_{n}$, so we have $n^{2} \equiv D_{1} D_{2}$ $(\bmod 4 p N)$. Therefore by summing $(7.17)$ over all pairs $\left(b_{1}, b_{2}\right)$ we get

$$
\left\langle\mathcal{P}_{D_{1}} \cdot \mathcal{P}_{D_{2}}\right\rangle_{\mathcal{X}}=2^{r-1} \cdot \eta(m) \cdot \sum_{p<\infty}\left(\sum_{\substack{n^{2}<D_{1} D_{2} \\ n^{2} \equiv D_{1} D_{2}(4 p N)}}\left(\sum_{\mathcal{E} \in \mathcal{C}_{p}} \frac{r_{\mathcal{E}}(n)}{u_{\mathcal{E}} v_{\mathcal{E}}}\right) \cdot \alpha_{p}\left(Q_{n}\right)\right) \cdot \log p .
$$

Let $\mathcal{E} \in \mathcal{C}_{p}$ and let $n$ be an integer such that $n^{2}<D_{1} D_{2}$ and $n^{2} \equiv D_{1} D_{2}$ $(\bmod 4 p N)$. Recall that $S_{n}=\mathbb{Z}\left[g_{1}, g_{2}\right]$ and that $L_{n}=\mathbb{Z}+\mathbb{Z} g_{1}+\mathbb{Z} g_{2} \subset S_{n}$ is a quadratic space with the reduced norm form $\operatorname{Nr}$. Let $(\tau, \lambda)$ be a pair consisting of a ring homomorphism $\tau: S_{n} \rightarrow \mathcal{E}$ and a unit $\lambda \in \mathcal{E}^{\times}$. Associated to this pair is an isometry $\left.\lambda \cdot \tau\right|_{L_{n}}$ of $L_{n}$ into $\mathcal{E}$. We claim that every isometry from $L_{n}$ into $\mathcal{E}$ arises this way. Let $\nu: L_{n} \rightarrow \mathcal{E}$ be an isometry, and set $\lambda=\nu(1)$. Then $1=\operatorname{Nr}(1)=\operatorname{Nr}(\lambda)$, so $\lambda \in \mathcal{E}^{\times}$. Let $\tau: S_{n} \rightarrow \mathcal{E}$ be the unique $\mathbb{Z}$-linear map such 
that $\tau(1)=1, \tau\left(g_{j}\right)=\lambda^{-1} \nu\left(g_{j}\right)$, and $\tau\left(g_{1} g_{2}\right)=\tau\left(g_{1}\right) \tau\left(g_{2}\right)$, and set $\epsilon_{j}=\tau\left(e_{j}\right)$ for $j=1,2$. Since $\nu$ is an isometry we get $\epsilon_{j}^{2}=-\operatorname{Nr}\left(\epsilon_{j}\right)=-\operatorname{Nr}\left(e_{j}\right)=D_{j}$ and

$$
\begin{aligned}
\left(\epsilon_{1}+\epsilon_{2}\right)^{2} & =-\operatorname{Nr}\left(\epsilon_{1}+\epsilon_{2}\right) \\
& =-\operatorname{Nr}\left(e_{1}+e_{2}\right) \\
& =\left(e_{1}+e_{2}\right)^{2},
\end{aligned}
$$

which implies $\epsilon_{1} \epsilon_{2}+\epsilon_{2} \epsilon_{1}=2 n$. It follows that

$$
\tau \otimes \mathbb{Q}: S_{n} \otimes \mathbb{Q} \longrightarrow \mathcal{E} \otimes \mathbb{Q}
$$

is an isomorphism of rings. Hence $\tau$ is a ring homomorphism such that $\nu=\left.\lambda \cdot \tau\right|_{L_{n}}$.

Since $\mathbb{Z}^{3}$ with the quadratic form $Q_{n}$ is isometric to $L_{n}$, it follows from the preceding paragraph that $\mathcal{R}_{\mathcal{E}}\left(Q_{n}\right)=\# \mathcal{E}^{\times} \cdot r_{\mathcal{E}}(n)=2 u_{\mathcal{E}} r_{\mathcal{E}}(n)$. By Lemma 5.3, every proper self-isometry of $\mathcal{E}$ has the form $x \mapsto u b x b^{-1}$ for some $u \in \mathcal{E}^{\times}$and $b \in N(\mathcal{E}) / \mathbb{Q}^{\times}$. Furthermore, $u$ and $b$ are uniquely determined by the isometry. Therefore the number of proper self-isometries of $\mathcal{E}$ is

$$
\begin{aligned}
w_{\mathcal{E}} & =\left|\mathcal{E}^{\times}\right| \cdot\left|N(\mathcal{E}) / \mathbb{Q}^{\times}\right| \\
& =\left|\mathcal{E}^{\times}\right| \cdot\left|N(\mathcal{E}) / \mathbb{Q}^{\times} \mathcal{E}^{\times}\right| \cdot\left|\mathbb{Q}^{\times} \mathcal{E}^{\times} / \mathbb{Q}^{\times}\right| \\
& =2 u_{\mathcal{E}} \cdot v_{\mathcal{E}} \cdot u_{\mathcal{E}} .
\end{aligned}
$$

Hence $r_{\mathcal{E}}(n) / u_{\mathcal{E}} v_{\mathcal{E}}=\mathcal{R}_{\mathcal{E}}\left(Q_{n}\right) / w_{\mathcal{E}}$, so by Proposition 5.2 we have

$$
\sum_{\mathcal{E} \in \mathcal{C}_{p}} \frac{r_{\mathcal{E}}(n)}{u_{\mathcal{E}} v_{\mathcal{E}}}=\sum_{L \in \mathcal{G}_{p}} \frac{\mathcal{R}_{L}\left(Q_{n}\right)}{w_{L}} .
$$

Substituting this formula into (7.18) gives Theorem 3.2.

Proof of Theorem 3.6: Let $n$ be such that $n^{2}<D_{1} D_{2}$ and $n \equiv h(\bmod 2 N)$. Since $\operatorname{gcd}\left(D_{1}, D_{2}\right)=1$, it follows from $\S 1$ that $S_{n}$ is an Eichler order of type $\left(\delta_{n}^{+}, \delta_{n}^{-}\right)$, and it follows from Remark 3.7 that $N^{+} \mid \delta_{n}^{+}$and $N^{-} \mid \delta_{n}^{-}$. Set $M_{n}^{+}=$ $\delta_{n}^{+} / N^{+}, M_{n}^{-}=\delta_{n}^{-} / N^{-}, m_{p}^{+}=v_{p}\left(M_{n}^{+}\right)$, and $m_{p}^{-}=v_{p}\left(M_{n}^{-}\right)$, and define $L_{(p)}(s)=$ $L_{p^{m_{p}^{+}}, p^{m_{p}^{-}}}(s)$. Then we have

$$
\begin{aligned}
& L_{M_{n}^{+}, M_{n}^{-}}(s)=\prod_{p<\infty} L_{(p)}(s), \\
& L_{M_{n}^{+}, M_{n}^{-}}^{\prime}(0)=\sum_{p<\infty}\left(\prod_{l \neq p} L_{(l)}(0)\right) L_{(p)}^{\prime}(0) .
\end{aligned}
$$


Let $p$ be a prime, and assume for now that $S_{n} \otimes \mathbb{Q} \cong \Delta(p)$. If $p \mid N^{-}$then $p \mid \delta_{n}^{-}$since $N^{-} \mid \delta^{-}$, while if $p \nmid N^{-}$then $p \mid \delta_{n}^{-}$since $p$ is ramified in $\Delta(p)$. Thus $p \nmid \delta^{+}$, and in particular $p \nmid N^{+}$. Let $\mathcal{E}_{0}$ be an Eichler order of type $\left(N^{+}, p N^{-}\right)$. We may compute $r_{\mathcal{E}_{0}}(n)$ as the number of embeddings of $\mathcal{E}_{0}$ into $S_{n} \otimes \mathbb{Q}$ whose image contains $S_{n}$. Hence $r_{\mathcal{E}_{0}}(n)$ is the sum over all $\mathcal{E}$ such that $S_{n} \subset \mathcal{E} \subset S_{n} \otimes \mathbb{Q}$ of the number of isomorphisms of $\mathcal{E}_{0}$ with $\mathcal{E}$. If $\mathcal{E}_{0} \cong \mathcal{E}$ then there are $\left|N\left(\mathcal{E}_{0}\right) / \mathbb{Q}^{\times}\right|=u_{\mathcal{E}_{0}} v_{\mathcal{E}_{0}}$ such isomorphisms. We conclude that the number of Eichler orders $\mathcal{E}$ such that $S_{n} \subset \mathcal{E} \subset S_{n} \otimes \mathbb{Q}$ and $\mathcal{E} \cong \mathcal{E}_{0}$ is $r_{\mathcal{E}_{0}}(n) / u_{\mathcal{E}_{0}} v_{\mathcal{E}_{0}}$. It follows that the inner sum $\sum_{\mathcal{E} \in \mathcal{C}_{p}} r_{\mathcal{E}}(n) / u_{\mathcal{E}} v_{\mathcal{E}}$ of $(7.17)$ is equal to the number of Eichler orders $\mathcal{E}$ of type $\left(N^{+}, p N^{-}\right)$such that $S_{n} \subset \mathcal{E} \subset S_{n} \otimes \mathbb{Q}$.

For each prime $l$ set $e_{l}^{+}=v_{l}\left(N^{+}\right), e_{l}^{-}=v_{l}\left(p N^{-}\right), d_{l}^{+}=v_{l}\left(\delta_{n}^{+}\right)$, and $d_{l}^{-}=$ $v_{l}\left(\delta_{n}^{-}\right)$. The number of Eichler orders in $S_{n} \otimes \mathbb{Q}$ of type $\left(N^{+}, p N^{-}\right)$which contain the Eichler order $S_{n}$ can be computed as the product over $l$ of the number $c_{l}$ of local Eichler orders $\mathcal{E}_{l}$ of type $\left(l_{l}^{e_{l}^{+}}, l_{l}^{e_{l}^{-}}\right)$in $S_{n} \otimes \mathbb{Q}_{l}$ such that $\mathcal{E}_{l} \supset S_{n} \otimes \mathbb{Z}_{l}$. If $S_{n} \otimes \mathbb{Z}_{l}$ has type $\left(l^{d_{l}^{+}}, 1\right)$ then $c_{l}=d_{l}^{+}-e_{l}^{+}+1=L_{(l)}(0)$. If $S_{n} \otimes \mathbb{Z}_{l}$ has type $\left(1, l_{l}^{d_{l}^{-}}\right)$then $c_{l}=1$ for $d_{l}^{-} \equiv e_{l}^{-}(\bmod 2)$ and $c_{l}=0$ for $d_{l}^{-} \not \equiv e_{l}^{-}(\bmod 2)$, so once again we have $c_{l}=L_{(l)}(0)$. In particular, since $S_{n} \otimes \mathbb{Q} \cong \Delta(p)$ we see that $S_{n} \otimes \mathbb{Z}_{p}$ has type $\left(1, p^{d_{p}^{-}}\right)$with $d_{p}^{-} \equiv e_{p}^{-}(\bmod 2)$. Hence $c_{p}=1$. It follows that the total number of Eichler orders $\mathcal{E}$ of type $\left(N^{+}, p N^{-}\right)$in $S_{n} \otimes \mathbb{Q}$ which contain $S_{n}$ is

$$
\sum_{\mathcal{E} \in \mathcal{C}_{p}} \frac{r_{\mathcal{E}}(n)}{u_{\mathcal{E}} v_{\mathcal{E}}}=\prod_{l \neq p} L_{(l)}(0) .
$$

Now suppose that $S_{n} \otimes \mathbb{Q}$ is not isomorphic to $\Delta(p)$. In this case the sum on the left side of $(7.29)$ is 0 , and there is at least one prime $l \neq p$ such that $v_{l}\left(M_{n}^{-}\right)$is odd. It follows that $L_{(l)}(0)=0$, so the right side of (7.29) is also 0. Hence (7.29) is valid for all primes $p$.

Assume once again that $S_{n} \otimes \mathbb{Q} \cong \Delta(p)$. If $p \nmid N$ then the assumption $\operatorname{gcd}\left(D_{1}, D_{2}\right)=1$ implies that, in the notation of (6.2), we have $a_{2}=0$ and $a_{3}=v_{p}\left(\delta_{n}\right)=v_{p}\left(\delta_{n}^{-}\right)$. Therefore $\alpha_{p}\left(Q_{n}\right)=\left(v_{p}\left(\delta_{n}^{-}\right)+1\right) / 2$. On the other hand, if $p \mid N^{-}$then by (6.6) we have $\alpha_{p}\left(Q_{n}\right)=v_{p}\left(\delta_{n}^{-}\right) / 2$. In both cases we get $\alpha_{p}\left(Q_{n}\right)=\left(v_{p}\left(M_{n}^{-}\right)+1\right) / 2$. Thus by (3.7) we get

$$
\begin{aligned}
L_{(p)}^{\prime}(0) & =\frac{v_{p}\left(M_{n}^{-}\right)+1}{2} \cdot \log p \\
& =\alpha_{p}\left(Q_{n}\right) \cdot \log p .
\end{aligned}
$$


It follows from (7.28), (7.29), and (7.31) that

$$
L_{M_{n}^{+}, M_{n}^{-}}^{\prime}(0)=\sum_{p<\infty}\left(\sum_{\mathcal{E} \in \mathcal{C}_{p}} \frac{r_{\mathcal{E}}(n)}{u_{\mathcal{E}} v_{\mathcal{E}}}\right) \cdot \alpha_{p}\left(Q_{n}\right) \cdot \log p .
$$

Combining this formula with (7.17) we get

$$
\left\langle\mathcal{P}_{D_{1}, \pm b_{1}} \cdot \mathcal{P}_{D_{2}, \pm b_{2}}\right\rangle_{\mathcal{X}}=2 \eta(m) \cdot \sum_{\substack{n^{2}<D_{1} D_{2} \\ n \equiv h(2 N)}} L_{M_{n}^{+}, M_{n}^{-}}^{\prime}(0),
$$

which is Theorem 3.6.

\section{REFERENCES}

[Bi] B. J. Birch, Heegner points of elliptic curves, Symposia Mathematica 15 (1975), 441-445.

[BC] J.-F. Boutot and H. Carayol, Uniformization $p$-adique des courbes de Shimura: les théorèmes de Cerednik et de Drinfeld, Astérisque 196-197 (1991), pp. 45-158.

[Bu] K. Buzzard, Integral models of certain Shimura curves, Duke Math. J. 87 (1997), 591612 .

[Ca] J. W. S. Cassels, Rational Quadratic Forms, Cambridge University Press (1978).

[Dr] V. G. Drinfeld, Coverings of $p$-adic symmetric regions (Russian), Funkcional. Anal. i Prilozen. 10 (1976) no. 2, 29-40; translated in Funct. Anal. Appl. 10 (1976), 107-115.

[Gr] B. Gross, On canonical and quasi-canonical liftings, Invent. Math. 84 (1986), 321-326.

[GK] B. Gross and K. Keating, On the intersection of modular correspondences, Invent. Math. 112 (1993), 225-245.

[GKZ] B. Gross, W. Kohnen, and D. Zagier, Heegner points and derivatives of $L$-series. II, Math. Ann. 278 (1987), 497-562.

[GZ1] B. Gross and D. Zagier, Heegner points and derivatives of L-series, Invent. Math. 84 (1986), 225-320.

[GZ2] B. Gross and D. Zagier, On singular moduli, J. Reine Angew. Math 355 (1985), 191-220.

[KM] N. Katz and B. Mazur, Arithmetic Moduli of Elliptic Curves, Princeton University Press (1985).

[Ke1] K. Keating, Lifting endomorphisms of formal $A$-modules, Compositio Math. 67 (1988), 211-239.

[Ke2] K. Keating, Intersection numbers of Heegner divisors on Shimura curves, preprint (1994).

$[\mathrm{Ku}] \quad$ S. Kudla, Central derivatives of Eisenstein series and height pairings, Ann. of Math. 146 (1997), 545-646.

[KR] S. Kudla and M. Rapoport, Height pairings on Shimura curves and $p$-adic uniformization, Invent. Math. 142 (2000), 153-223.

[KRY] S. Kudla, M. Rapoport, and T. Yang, Modular forms and special cycles on Shimura curves, Princeton University Press (2006). 
[LT] J. Lubin and J. Tate, Formal moduli for one-parameter formal Lie groups, Bull. Soc. Math. France 94 (1966), 49-59.

[Mi] J. Milne, Étale Cohomology, Princeton University Press (1980).

[Oo] F. Oort, Endomorphism algebras of abelian varieties, appearing in Algebraic geometry and commutative algebra: in honor of Masayoshi Nagata, Academic Press (1988), 469502.

[Re] I. Reiner, Maximal Orders, Academic Press (1975).

[Ro] D. P. Roberts, Shimura curves analogous to $X_{0}(N)$, Harvard Ph.D. Thesis, 1989.

[Sha] I. Shafarevich, Lectures on Minimal Models and Birational Transformations, Tata Institute (1966).

[Shi] T. Shioda, Supersingular K3 surfaces, Lecture Notes in Math. 732 (1979), 564-591.

[Te] J. Teitelbaum, On Drinfeld's universal formal group over the $p$-adic upper half plane, Math. Ann. 284 (1989), 647-674.

[Vi] M. Vignéras, Arithmétique des Algèbres de Quaternions, Lecture Notes in Math. 800 (1980).

[Zh] S.-W. Zhang, Heights of Heegner points on Shimura curves, Ann. of Math. (2) 153 (2001), 27-147.

Kevin Keating

Department of Mathematics

University of Florida

Gainesville, FL 32611

USA

E-mail: keating@math.ufl.edu

David P. Roberts

Division of Science and Mathematics

University of Minnesota

Morris, MN 56267

USA

E-mail: roberts@morris.umn.edu 